\title{
Bound states and entanglement in the excited states of quantum spin chains
}

\author{
Jan Mölter ${ }^{1}$, Thomas Barthel ${ }^{2,1}$, Ulrich Schollwöck ${ }^{1}$, and \\ Vincenzo Alba ${ }^{1}$ \\ ${ }^{1}$ Department of Physics and Arnold Sommerfeld Center for Theoretical Physics, \\ Ludwig-Maximilians-Universität München, D-80333 München, Germany \\ ${ }^{2}$ Laboratoire de Physique Théorique et Modèles statistiques, Université Paris-Sud \\ and CNRS, UMR 8626, 91405 Orsay, France
}

\begin{abstract}
.
We investigate entanglement properties of the excited states of the spin- $\frac{1}{2}$ Heisenberg $(X X X)$ chain with isotropic antiferromagnetic interactions, by exploiting the Bethe ansatz solution of the model. We consider eigenstates obtained from both real and complex solutions ("strings") of the Bethe equations. Physically, the former are states of interacting magnons, whereas the latter contain bound states of groups of particles. We first focus on the situation with few particles in the chain. Using exact results and semiclassical arguments, we derive an upper bound $S_{M A X}$ for the entanglement entropy. This exhibits an intermediate behavior between logarithmic and extensive, and it is saturated for highly-entangled states. As a function of the eigenstate energy, the entanglement entropy is organized in bands. Their number depends on the number of blocks of contiguous Bethe-Takahashi quantum numbers. In presence of bound states a significant reduction in the entanglement entropy occurs, reflecting that a group of bound particles behaves effectively as a single particle. Interestingly, the associated entanglement spectrum shows edge-related levels. At finite particle density, the semiclassical bound $S_{M A X}$ becomes inaccurate. For highlyentangled states $S_{A} \propto L_{c}$, with $L_{c}$ the chord length, signaling the crossover to extensive entanglement. Finally, we consider eigenstates containing a single pair of bound particles. No significant entanglement reduction occurs, in contrast with the fewparticle case.
\end{abstract}




\section{Introduction}

In recent years the study of entanglement measures and other entanglement-related quantities have become of paramount importance in order to understand the behavior of low-dimensional quantum many-body systems [1-4]. Considering a system $S$ in a pure state $|\Psi\rangle$, and its spatial bipartition into two blocks $A$ and $B$, as $S=A \cup B$, one can decompose $|\Psi\rangle$ as (Schmidt decomposition)

$$
|\Psi\rangle=\sum_{i} e^{-\xi_{i} / 2}\left|\psi_{i}^{A}\right\rangle \otimes\left|\psi_{i}^{B}\right\rangle
$$

with $\left|\psi_{i}^{A}\right\rangle$ and $\left|\psi_{i}^{B}\right\rangle$ two orthonormal bases for the subsystems Hilbert spaces $\mathcal{H}_{A}$ and $\mathcal{H}_{B}$. Here the so-called entanglement spectrum (ES) levels $\left\{\xi_{i}\right\}[5-46]$ are related to the eigenvalues $\left\{\lambda_{i}\right\}$ of the reduced density matrix for part $A, \rho_{A} \equiv \operatorname{Tr}_{B}(|\Psi\rangle\langle\Psi|)$, as $\xi_{i} \equiv-\log \left(\lambda_{i}\right)$. A proper measure of the quantum entanglement between $A$ and $B$ is the so-called von Neumann entropy (entanglement entropy) $S_{A}$, which is defined as

$$
S_{A} \equiv-\sum_{i} \lambda_{i} \log \left(\lambda_{i}\right)
$$

It is now well established that the ground-state entanglement entropy contains universal information about one-dimensional quantum many-body systems [1-3]. A spectacular example is provided by critical systems described by a conformal field theory (CFT), for which it has been proven that $S_{A}$ obeys the scaling behavior [47-49]

$$
S_{A}=\frac{c}{3} \log \left[\frac{L}{\pi} \sin \left(\frac{\pi L_{A}}{L}\right)\right]+c_{1}^{\prime} .
$$

Here $c$ is the central charge of the conformal field theory, $c_{1}^{\prime}$ a non-universal constant, while $L$ and $L_{A}$ are the sizes of the full system and of part $A$, respectively. Universal information can also be extracted from the entanglement between many disjoint blocks [50-62], or from the scaling corrections of the von Neumann entropy [63-69].

In contrast, although many results are available in the literature [70-82], much less is known about entanglement properties of excited states. Remarkably, for eigenstates obtained from primary fields of a CFT, which correspond to low-energy excitations, a scaling law similar to (3) has been obtained in Ref. [71,73]. Some results are available for exactly-solvable and free models. A complete classification of entanglement behaviors in the excited states of the $X X$ chain [83-87] is provided in Ref. [70] (see also [80]). Two main classes of eigenstates are present, displaying logarithmic (similar to (3), with an effective "central charge"), or the extensive behavior $S_{A} \propto L_{A}$, respectively. The two scenarios are related to the local properties of the eigenstates, namely the distribution of occupied momenta in the Fermi sea that is obtained after mapping the $X X$ chain onto a free-fermion model. Interestingly, a logarithmic entanglement scaling is also observed in some high-energy eigenstates of the $X X Z$ chain. This has been shown in Ref. [70] using a numerical method based on the algebraic Bethe ansatz solution of the model [88]. Yet, due to technical reasons, Ref. [70] focuses only on eigenstates obtained from real solutions of the so-called Bethe equations, and for small anisotropy (i.e., near the non-interacting $X X$ chain limit). 
Although it is interesting per se, the study of entanglement in excited states arises naturally in the context of out-of-equilibrium dynamics after a quantum quench. Formally, at any time after the quench the state of the system can be obtained as a time-dependent superposition of the eigenstates of the post-quench Hamiltonian. Understanding entanglement properties in individual eigenstates could be helpful in devising an effective truncation scheme to study real-time dynamics, especially in integrable models $[89,90]$. On the numerical side, this could give insights on the simulability of quench problems using DMRG (Density Matrix Renormalization Group) or other MPS (Matrix Product States) based algorithms [91-95]. Similarly, as excited states are necessary ingredients in constructing the Gibbs ensemble, a detailed knowledge of their entanglement properties could be useful for studying finite-temperature systems.

Outline of the results. In this paper we investigate the entanglement entropy of the eigenstates of the spin- $\frac{1}{2}$ isotropic Heisenberg $(X X X)$ chain. This is defined by the Hamiltonian

$$
\mathcal{H}=J \sum_{i=1}^{L} \frac{1}{2}\left(S_{i}^{+} S_{i+1}^{-}+S_{i}^{-} S_{i+1}^{+}\right)+S_{i}^{z} S_{i+1}^{z},
$$

where $S_{i}^{+,-, z}$ are the raising and lowering spin operators $S_{i}^{ \pm} \equiv\left(\sigma_{i}^{x} \pm i \sigma_{i}^{y}\right) / 2, S_{i}^{z} \equiv \sigma_{i}^{z} / 2$, and $\sigma_{i}^{x, y, z}$ the Pauli matrices. We set for convenience $J=1$ in (4). Periodic boundary conditions are used, i.e., sites $L+1$ and 1 are identified. The $X X X$ chain is exactly solvable, and its eigenstates and eigenenergies can be constructed via the Bethe ansatz $[88,96,97]$. The Hilbert space of (4) is spanned by $2^{L}$ basis states, which can be generated starting from the ferromagnetic state $|\Omega\rangle \equiv|\uparrow \uparrow \uparrow \cdots \uparrow\rangle$, flipping $M$ of the spins, with $M \in[0, L]$. Since it is conventional in the Bethe ansatz literature, we refer to flipped spins as "particles". The total magnetization $S_{T}^{z} \equiv \sum_{i=1}^{L} S_{i}^{z}=L / 2-M$ is a conserved quantity for (4), implying that the eigenstates of the $X X X$ chain can be labeled by $S_{T}^{z}$ (equivalently by $M$ ).

In contrast with Ref. [70], here we consider eigenstates of the $X X X$ chain obtained from both real and complex ("strings") solutions of the Bethe equations (rapidities). Physically, while the former correspond to interacting magnons, complex rapidities signal the presence of many-particle bound states. Notice that the intriguing physics of bound states has received constant attention, both theoretically [98-103] and experimentally [104, 105]. Finally, while Ref. [70] focuses mostly on eigenstates of the $X X Z$ chain exhibiting logarithmic entanglement entropy, here we characterize the crossover to extensive entanglement, which is expected in highly-entangled eigenstates of $(4)$.

We first focus on the situation with few particles in the chain, restricting ourselves to $M=2$ and $M=3$, i.e., high-magnetization sectors of (4). For any eigenstate in the two-particle sector $(M=2)$, its entanglement entropy and entanglement spectrum are constructed analytically exploiting the Bethe ansatz solution of (4). This, in particular, allows us to derive an upper bound $S_{M A X}$ for the entanglement entropy. 
Its generalization to arbitrary $M$ is obtained using semiclassical arguments as

$$
S_{M A X}(M)=-M(\omega \log (\omega)+(1-\omega) \log (1-\omega)),
$$

where $\omega$ is the ratio $\omega \equiv L_{A} / L$. Clearly, for $M=1, S_{M A X}$ is the entanglement entropy of a free particle. For $M=2$ the bound (5) has been also discussed in Ref. [76]. The linear dependence $\propto M$ in (5) reflects that in the semiclassical approximation the interactions (scattering) between particles can be neglected, as expected. Interestingly, for $L_{A} / L \ll 1$ (small blocks) one has $S_{M A X} \approx-\left(M L_{A} / L\right) \log \left(L_{A} / L\right)$, which is an "intermediate" behavior between a logarithmic and an extensive growth. Notice that (5) is similar to the entanglement entropy of a ferromagnet [106-109]. In the sector with $M=2$ and for real rapidities we find that $S_{M A X}(M)$ is saturated for any $L_{A}$, meaning that $S_{A} \approx S_{M A X}$ (apart from $\mathcal{O}(1 / L)$ terms) for some highly-entangled eigenstates of (4).

We also investigate the behavior of the half-chain entanglement entropy as a function of the eigenstate energy. For $M=2$ and real rapidities, a two-band structure appears, with eigenstates in the same band showing similar entanglement. In the higher band we have $S_{A} \approx S_{M A X}$. This band structure is understood in terms of the two BetheTakahashi quantum numbers, which in the Bethe ansatz formalism are used to identify the eigenstates of (4) [97]. Specifically, the lower and upper band correspond to the two quantum numbers being next to each other or far apart, respectively.

For states with real rapidities only, these features are generic in the vanishing density regime $M / L \rightarrow 0$ at fixed $M$ : In practice, we numerically observe that the bound (5) is almost saturated. Moreover, the entanglement entropy is organized in bands. Higher entanglement bands correspond to increasing number of blocks of contiguous BetheTakahashi quantum numbers. For a given $M$, the number of bands is hence obtained as the number of integer partitions $p(M)$ of $M$. Physically, a small (large) number of blocks of Bethe-Takahashi numbers reflects the particles having small (large) relative quasi-momentum. All these findings are numerically confirmed by studying the full set of eigenstates of (4) (for finite $L$ ) in the sector with $M=3$.

The scenario is strikingly different in presence of bound states. For bound states of two particles (2-string solutions of the Bethe equations) a substantial decrease in the entanglement entropy is observed, as compared with eigenstates obtained from two real rapidities. This reflects a reduction in the number of degrees of freedom, since the bound state can be treated as an effective single particle. Nevertheless, we observe that $S_{A}>S_{M A X}(M=1)$, signaling that the bound states are extended objects. This is quantitatively understood in terms of the bound-state extension $\ell_{b}$, which is obtained analytically from the Bethe ansatz solution of (4), and is an increasing function of the bound-state energy. As a consequence, while high-energetic bound states with $\ell_{b} / L \approx 1$ behave almost as two unbound particles, low-energy ones with $\ell_{b} / L \ll 1$ can be treated as a single particle. Clearly, larger values of $\ell_{b} \lesssim L$ are associated with higher entanglement. Interestingly, since in the large $L$ limit $\ell_{b}$ changes continuously as a function of the bound-state energy, no bands are observed in the entanglement 
entropy, in contrast with the case with two real rapidities.

The presence of bound states has striking effects also at the level of the half-chain entanglement spectrum (ES). Specifically, we show that the ES exhibits edge-related levels. The corresponding entanglement eigenfunctions (i.e., the eigenvectors of the reduced density matrix) are exponentially localized at the boundary between the two subsystems (edge-states). Similar features, i.e., reduction of the entanglement entropy, edge-related ES levels, and absence of entanglement bands, are observed at fixed $M>2$, in the limit $L \rightarrow \infty$.

This scenario breaks down at finite particle density $\rho \equiv M / L$. A striking change is that the upper bound (5) is no longer saturated. However, we numerically observe that in highly-entangled eigenstates $S_{A} \propto L_{c}$, where $L_{c}=(L / \pi) \sin \left(\pi L_{A} / L\right)$ is the so-called chord length. Since $L_{c} \approx L_{A}$ for $L_{A} \ll L$, this signals the crossover from (5) to the volume law $S_{A} \propto L_{A}$. We also investigate how the entanglement entropy depends on the Bethe-Takahashi quantum numbers. Similarly to the situation with few particles, we find that a large number of Bethe number blocks tends to correspond to highly-entangled eigenstates of the $X X X$ chain. Finally, we focus on the eigenstates with $\rho \approx 1 / 2$ containing a single two-particle bound state. We show that no significant entanglement reduction occurs (at least for finite chains), in contrast with the few-particle case.

\section{Survey of Bethe ansatz results for the $X X X$ chain}

We start reviewing some aspects of the Bethe ansatz solution of (4) (see $[88,96,97,110]$ for more details) that are relevant for this work. In section 2.1 the generic form of the eigenstates of (4) and the so-called Bethe equations are introduced. Their solutions, both real (1-strings) and complex ( $n$-strings, with $n \in \mathbb{N}, n>1$ ), are discussed in 2.2 and 2.3, within the framework of the string hypothesis [97]. In particular, we introduce the so-called Bethe-Takahashi equations. Since it is important in order to understand entanglement properties of the $X X X$ chain, we detail the structure of the Bethe-Takahashi quantum numbers, which identify the solutions of the Bethe-Takahashi equations, and the eigenstates of (4) thereof. Finally, focusing on complex solutions of the Bethe-Takahashi equations, we define the bound state length $\ell_{b}$.

\subsection{Bethe equations and wavefunctions}

The generic eigenstate of (4) in the sector with $M$ particles can be written as

$$
\left|\Psi_{M}\right\rangle=\sum_{1 \leq x_{1}<x_{2}<\ldots<x_{M} \leq L} A_{M}\left(x_{1}, x_{2}, \ldots, x_{M}\right)\left|x_{1}, x_{2}, \ldots, x_{M}\right\rangle,
$$

where the sum is over the positions $\left\{x_{i}\right\}$ of the particles, and $A_{M}\left(x_{1}, x_{2}, \ldots, x_{M}\right)$ is the eigenstate amplitude corresponding to particles at positions $x_{1}, x_{2}, \ldots, x_{M}$. $A_{M}\left(x_{1}, x_{2}, \ldots, x_{M}\right)$ is given as

$$
A_{M}\left(x_{1}, x_{2}, \ldots, x_{M}\right) \equiv \sum_{\mathcal{P} \in S_{M}} \exp \left[i \sum_{j=1}^{M} k_{\mathcal{P}_{j}} x_{j}+i \sum_{i<j} \theta_{\mathcal{P}_{i} \mathcal{P}_{j}}\right] .
$$


Here the outermost sum is over the permutations $S_{M}$ of the so-called quasi-momenta $\left\{k_{1}, k_{2}, \ldots, k_{M}\right\}$. The two-particle scattering phases $\theta_{m, n}$ are defined as

$$
\theta_{m, n} \equiv \frac{1}{2 i} \log \left[-\frac{e^{i k_{m}+i k_{n}}-2 e^{i k_{m}}+1}{e^{i k_{m}+i k_{n}}-2 e^{i k_{n}}+1}\right] .
$$

The energy associated to the eigenstate (6) is

$$
E=\sum_{\alpha=1}^{M}\left(\cos \left(k_{\alpha}\right)-1\right) \text {. }
$$

The quasi-momenta $\left\{k_{\alpha}\right\}$ are obtained by solving the so-called Bethe equations

$$
e^{i k_{\alpha} L}=\prod_{\beta \neq \alpha}^{M}\left[-\frac{1-2 e^{i k_{\alpha}}-e^{i k_{\alpha}+i k_{\beta}}}{1-2 e^{i k_{\beta}}-e^{i k_{\alpha}+i k_{\beta}}}\right] .
$$

It is useful to introduce the rapidities $\left\{\lambda_{\alpha}\right\}$ as

$$
k_{\alpha}=\pi-2 \arctan \left(\lambda_{\alpha}\right) \bmod 2 \pi .
$$

Taking the logarithm on both sides in (10), and using (11), one obtains the Bethe equations in logarithmic form as

$$
\arctan \left(\lambda_{\alpha}\right)=\frac{\pi}{L} J_{\alpha}+\frac{1}{L} \sum_{\beta \neq \alpha} \arctan \left(\frac{\lambda_{\alpha}-\lambda_{\beta}}{2}\right),
$$

where $-L / 2<J_{\alpha} \leq L / 2$ are the so-called Bethe quantum numbers. Notice that $J_{\alpha}$ are half-integers and integers for $L-M$ even and odd, respectively [97]. Finally, the total eigenstate quasi-momentum $K_{T} \equiv \sum_{\alpha} k_{\alpha}$ is obtained from (12) and (11) as

$$
K_{T}=\pi M+\frac{2 \pi}{L} \sum_{\alpha} J_{\alpha}
$$

\subsection{Real roots of the Bethe equations (1-strings)}

In principle any choice of $M$ distinct Bethe quantum numbers $\left\{J_{1}, J_{2}, \ldots, J_{M}\right\}$ identifies a set of solutions $\left\{k_{1}, k_{2}, \ldots, k_{M}\right\}$ of (12) and, using (6), an eigenstate of (4). However, for real solutions of (12), i.e. $\lambda_{\alpha} \in \mathbb{R} \forall \alpha$, a precise bound for the Bethe numbers can be obtained as [97]

$$
-J_{\infty} \leq J_{\alpha} \leq J_{\infty} \quad \text { with } \quad J_{\infty} \equiv \frac{L-1-M}{2}
$$

where $J_{\infty}$ is formally the Bethe quantum number associated to an infinite rapidity [97]. It is convenient to introduce the notation - and $\bigcirc$ for occupied and vacant ("holes") Bethe quantum numbers, respectively. A generic eigenstate of (4), in the sector with $M$ particles, can then be identified by its Bethe quantum numbers as

$$
\left[\bigcirc^{m_{1}} \bigcirc^{n_{1}} \bigcirc^{m_{2}} \mathbf{\bigcirc}^{n_{2}} \cdots\right]_{1} \quad \text { with } \mathbf{\circ}^{m} \equiv \underbrace{\mathbf{0} \ldots \boldsymbol{\bullet}}_{m}
$$

where $\sum_{i} n_{i}=M$ and $\sum_{i} m_{i}=2 J_{\infty}+1-M$. The brackets $[\cdots]_{1}$ mean that the Bethe quantum numbers are defined in the interval $\left[-J_{\infty}, J_{\infty}\right]$, whereas the subscript is to 
stress that real solutions of (12) (1-strings, see section 2.3) are considered. The total number of real rapidities, according to (14), is $(L-M) ! /(M !(L-2 M) !)$.

Remarkably, the lowest-energy eigenstate $\left|\Psi_{M}^{(0)}\right\rangle$ of (4) in the sector with $M$ particles is given in terms of real rapidities [111], which are obtained by choosing the Bethe quantum numbers

$$
J_{\alpha}^{(0)}(M)=-\frac{M+1}{2}+\alpha \quad \text { with } \quad \alpha=1,2, \ldots, M,
$$

or, in the notation (15),

$$
J_{\alpha}^{(0)}(M)=\left[\bigcirc^{(L-M) / 2} \bigcirc^{M} \bigcirc^{(L-M) / 2}\right]_{1} .
$$

Notice that (14) implies that in the sector with $M=L / 2$ there is only one set of real roots of the Bethe equations, which corresponds to the global ground state $\left|\Psi^{(0)}\right\rangle$ of the $X X X$ chain.

It is natural to interpret the block " ${ }^{M}$ " of occupied Bethe numbers in (17) as a "Fermi sea". All the excited states above $\left|\Psi_{M}^{(0)}\right\rangle$, which have the same number of particles, and correspond to real rapidities, are obtained by successive exchanges of occupied and empty Bethe quantum numbers ("particle-hole" processes). An example of such processes is shown in Table 1 (first row, p-hole).

It is useful to classify the eigenstates of the $X X X$ chain according to the number of blocks of contiguous Bethe quantum numbers. While the ground state $\left|\Psi_{M}^{(0)}\right\rangle$ (cf. (17)) corresponds to a single block, the number of blocks generically increases under p-hole processes (for instance from one to three in the process shown in Table 1), although this is not true in general. A notable exception is provided by sequences of p-hole processes that amount to a shift of one or many blocks of quantum numbers. For instance, the process

$$
\left[\cdots \bigcirc \bigcirc^{M^{\prime}} \bigcirc^{h} \bigcirc^{M^{\prime \prime}} \bigcirc \cdots\right]_{1} \rightsquigarrow\left[\cdots \bigcirc \bigcirc^{M^{\prime}} \bigcirc^{h^{\prime}} \bigcirc^{M^{\prime \prime}} \bigcirc \cdots\right]_{1},
$$

where the dots stand for $\bigcirc \cdots \bigcirc, M^{\prime}+M^{\prime \prime}=M$, and $h \neq h^{\prime}$ are non-zero integers. The number of blocks in the Bethe quantum numbers is strikingly reflected in the entanglement properties of the excited states of the $X X X$ chain. We anticipate here that eigenstates obtained from Bethe quantum number configurations with the same number of blocks exhibit similar entanglement entropy (see section 3 and 4).

A different class of excited states of the Heisenberg chain is obtained from roots of (10) containing pairs of complex conjugate rapidities (strings), which, physically, correspond to groups of bound particles. An example of process leading to an eigenstate with a two-particle bound state is shown in Table 1 (see next section for the structure of the corresponding Bethe quantum numbers).

\subsection{String solutions of the Bethe equations}

Complex solutions of the Bethe equations (10) form particular "string" patterns in the complex plane, at least in the limit of large chains $L \rightarrow \infty$ (string hypothesis) [96,97]. 
Table 1. Excitations processes and Bethe-Takahashi quantum numbers for some eigenstates of the $X X X$ spin chain in the sector with $M$ particles. Only processes preserving the total number of particles are considered. Here $\bullet(\bigcirc)$ denotes an occupied (vacant) quantum number, $[\cdots]_{\alpha}$ is the quantum number configuration in the $\alpha$-string sector, and $s_{1} \equiv(L-M) / 2, s_{2} \equiv(L-2 M) / 2$, with $L$ the length of the chain. Notice that 1-strings correspond to real rapidities. $\left[\bigcirc^{s_{1}} \bigcirc^{M} \bigcirc^{s_{1}}\right]_{1}$ corresponds to the lowest-energy state in the sector with $M$ particles. (First row) p-hole excitation: one occupied and empty numbers are exchanged. An example of p-hole process is shown in the second column. The number of blocks of contiguous Bethe quantum numbers increases from one to three. All 1-strings excited states of the $X X X$ chain can be obtained by successive applications of p-hole. (Second row) Creation of a twoparticle bound state (2-string). Excited states involving $n$-strings with $n>2$, or more than a single bound states are not shown.

\begin{tabular}{ccc}
\hline Type of excitation & & Bethe-Takahashi numbers \\
\hline p-hole & {$\left[\bigcirc^{s_{1}} \bigcirc^{M} \bigcirc^{s_{1}}\right]_{1} \rightsquigarrow$} & {$\left[\bigcirc^{s_{1}} \bigcirc \bigcirc^{M-2} \bigcirc^{s_{1}-2} \bigcirc\right]_{1}$} \\
2-string & {$\left[\bigcirc^{s_{1}} \bigcirc^{M} \bigcirc^{s_{1}}\right]_{1} \rightsquigarrow$} & {$\left[\bigcirc^{s_{1}} \bigcirc^{M / 2-1} \bigcirc^{2} \bigcirc^{M / 2-1} \bigcirc^{s_{1}}\right]_{1}$} \\
& & {$\left[\bigcirc^{s_{2}} \bigcirc^{s_{2}}\right]_{2}$} \\
\hline
\end{tabular}

Specifically, rapidities forming a "string" of length $1 \leq n \leq M$ (that we defined here as $n$-string) are parametrized as

$$
\lambda_{\gamma}^{(n, j)}=\lambda_{\gamma}^{(n)}-i(n-1-2 j), \quad j=0,1, \ldots, n-1,
$$

where $\lambda_{\gamma}^{(n)}$ is the real part of the string (string center), and $\gamma$ labels strings with different centers. In this language real rapidities are strings of unit length (1-strings), i.e., with $n=1$. It is natural to classify solutions $\Lambda \equiv\left\{\lambda_{\alpha}\right\}(\alpha \in[1, M])$ of the Bethe equations (10) according to their string content $\left\{N_{\alpha}\right\}$, where $N_{\alpha}$ denotes the number of $\alpha$-strings in $\Lambda$.

Bethe-Takahashi equations. The string centers $\lambda_{\gamma}^{(n)}$ in (19) are obtained by solving the so-called Bethe-Takahashi equations [97]

$$
2 L \arctan \left(\lambda_{\gamma}^{(n)} / n\right)=2 \pi I_{\gamma}^{(n)}+\sum_{(m, \beta) \neq(n, \gamma)} \Theta_{m, n}\left(\lambda_{\gamma}^{(n)}-\lambda_{\beta}^{(m)}\right),
$$

where the generalized scattering phases $\Theta_{m, n}$ read

$$
\Theta_{m, n}(x) \equiv\left\{\begin{array}{cl}
\vartheta\left(\frac{x}{|n-m|}\right)+\sum_{r=1}^{(n+m-|n-m|-1) / 2} 2 \vartheta\left(\frac{x}{|n-m|+2 r}\right)+\vartheta\left(\frac{x}{n+m}\right) & \text { if } n \neq m \\
\sum_{r=1}^{n-1} 2 \vartheta\left(\frac{x}{2 r}\right)+\vartheta\left(\frac{x}{2 n}\right) & \text { if } n=m
\end{array}\right.
$$


and $\vartheta(x) \equiv 2 \arctan (x)$. Here $I_{\gamma}^{(n)}$ are the Bethe-Takahashi quantum numbers associated with $\lambda_{\gamma}^{(n)}$. It can be shown that $I_{\gamma}^{(n)}$ are integers or half-integers for $L-N_{n}$ odd and even, respectively. Clearly, the constraint $\sum_{\alpha=1}^{M} \alpha N_{\alpha}=M$ has to be satisfied. As for the Bethe quantum numbers $J_{\alpha}$ (see (14)), an upper bound for the Bethe-Takahashi quantum numbers can be derived as [97]

$$
\left|I_{\gamma}^{(n)}\right| \leq I_{M A X}^{(n)} \equiv \frac{1}{2}\left(L-1-\sum_{m=1}^{M} t_{m, n} N_{m}\right),
$$

where $t_{m, n} \equiv 2 \min (n, m)-\delta_{m, n}$. Notice that this implies $I_{M A X}^{(n)} \leq I_{M A X}^{\left(n^{\prime}\right)}$ if $n<n^{\prime}$.

It is straightforward to generalize the notation (15) to the case of the Bethe-Takahashi quantum numbers. First, for real solutions of the Bethe-Takahashi equations, i.e., $\lambda_{\alpha} \in \mathbb{R}, \forall \alpha$, one has $\left\{J_{\alpha}\right\}=\left\{I_{\alpha}^{(1)}\right\}$. Moreover, given a generic solution of (20) with string content $\left\{N_{\alpha}\right\}$, the corresponding Bethe-Takahashi numbers can be represented as $\left[\mathcal{C}_{1}\right]_{1} \times\left[\mathcal{C}_{2}\right]_{2} \times \cdots \times\left[\mathcal{C}_{M}\right]_{M}$, with

$$
\left[\mathcal{C}_{\alpha}\right]_{\alpha} \equiv\left[\bigcirc^{m_{1}} \bullet^{n_{1}} \bigcirc^{m_{2}} \bullet^{n_{2}} \cdots\right]_{\alpha}
$$

the Bethe-Takahashi numbers corresponding to the $N_{\alpha} \alpha$-strings. Notice that $\sum_{i} n_{i}=$ $N_{\alpha}$ and $\sum_{i} m_{i}=2 I_{M A X}^{(\alpha)}+1-N_{\alpha}$.

The quasi-momentum associated to a generic rapidity $\lambda^{\prime}=\lambda+i p$ in a $n$-string, using (11), is

$$
k(\lambda+i p)= \begin{cases}\pi+\frac{i}{2} \log \left[\frac{(1-p)^{2}+\lambda^{2}}{(1+p)^{2}+\lambda^{2}}\right]-\arctan \left[\frac{\lambda}{1+p}\right]-\arctan \left[\frac{\lambda}{1-p}\right] & \text { if } \quad|p|>1 \\ \pi+\frac{i}{2} \log \left[\frac{(1-p)^{2}+\lambda^{2}}{(1+p)^{2}+\lambda^{2}}\right]-\arctan \left[\frac{\lambda}{1+|p|}\right]+\frac{\pi}{2} \operatorname{Sign}(\lambda) & \text { if } \quad|p|=1,\end{cases}
$$

where $\operatorname{Sign}(x)$ is the sign function. By summing over the different string components, it is straightforward to obtain the total quasi-momentum $k_{s}^{(n)}$ of the $n$-string as

$$
k_{s}^{(n)}=\pi+2 \arctan \left(\frac{\lambda_{\gamma}^{(n)}}{n}\right) \bmod 2 \pi .
$$

The corresponding string energy $E_{s}^{(n)}$, from (9) and (23), is a function of the string center $\lambda_{\gamma}^{(n)}$ as

$$
E_{s}^{(n)}=-\frac{2 n}{\left(\lambda_{\gamma}^{(n)}\right)^{2}+n^{2}} .
$$

Interestingly, since $0<|\lambda|<\infty$ one has $E_{s}^{(n)} \in[-2 / n, 0]$, implying that "longer" strings have higher energy.

It is useful to isolate the imaginary part of the quasi-momentum $k(\lambda+i p)$ in (23), defining $\ell_{b}^{(p)}$ as

$$
\ell_{b}^{(p)} \equiv\left[\frac{1}{2} \log \frac{(1+p)^{2}+\lambda^{2}}{(1-p)^{2}+\lambda^{2}}\right]^{-1} .
$$

Since $\ell_{b}^{(p)}$ contributes with terms of the form $\exp \left(-\left|x_{i}-x_{j}\right| / \ell_{b}^{(p)}\right)$ in the amplitudes (7), it is natural to interpret $\ell_{b}^{(p)}$ as the bound-state extension. Large $\ell_{b}^{(p)}$ corresponds to 
a weakly-bound cluster of particles, whereas $\ell_{b}^{(p)} \rightarrow 0$ signals a strongly-bound one. Finally, we define $\ell_{b}$ as the length associated to the outermost rapidity in a string, i.e., that with the largest imaginary part. This can be expressed in terms of the string total energy $E_{s}^{(n)}$ (cf. (25)) as

$$
\ell_{b}=\left[-\frac{1}{2} \log \left(1+\frac{2 n-2}{n} E_{s}^{(n)}\right)\right]^{-1}
$$

Since in this work we restrict ourselves to strings with $n=2,3$, we consider only $\ell_{b}$.

We should stress that the string hypothesis (19) holds only in the limit $L \rightarrow \infty$, whereas for finite chains rapidities of the form (19) are not solutions of the Bethe equations (12). To account for finite chain corrections, Equation (19) has to be modified as (deviated $n$-string)

$$
\lambda_{\gamma}^{(n, j)}=\lambda_{\gamma}^{(n)}+\epsilon_{\gamma}^{(n, j)}+i(n-1-2 j)+i \delta_{\gamma}^{(n, j)} \quad \text { with } \quad j=0,1, \ldots, n-1,
$$

where the string deviations $\epsilon_{\gamma}^{(n, j)}, \delta_{\gamma}^{(n, j)} \in \mathbb{R}$ are $\mathcal{O}\left(e^{-L}\right)$, i.e., exponentially vanishing in the limit $L \rightarrow \infty$. One should remark that solutions of the Bethe equations deviating from the string picture (28) are known. For instance, for two-particle, they have been carefully characterized in [112] (cf. also [113]). Similar behaviors can be observed for open boundary conditions [114]. Furthermore, detailed knowledge of the string deviations is important in order to extract physical quantities (for instance conformal data) about the Heisenberg chain [115-119]. Notice that solutions of the Bethe equations are self-conjugate [120], i.e., $\left\{\lambda_{\alpha}\right\}=\left\{\lambda_{\alpha}\right\}^{*}$, which implies some constraints on the string deviations.

It is interesting to calculate the scattering phases (8) for deviated strings. Given $\lambda_{1}$ and $\lambda_{2}$ as $\lambda_{1}=\lambda+\epsilon+i p+i \delta$ and $\lambda_{2}=\lambda+\epsilon^{\prime}+i q+i \delta^{\prime}$, with $p, q \in \mathbb{Z}$ and $\epsilon, \epsilon^{\prime}, \delta, \delta^{\prime} \in \mathbb{R}$, one has using (8) and (28)

$$
\begin{aligned}
\theta\left(\lambda_{1}, \lambda_{2}\right)= & \frac{1}{4 i} \log \left[\frac{(p-q+2+\hat{\delta})^{2}+\hat{\epsilon}^{2}}{(p-q-2+\hat{\delta})^{2}+\hat{\epsilon}^{2}}\right]+ \\
& \frac{1}{2} \arctan \left[\frac{4 \hat{\epsilon}}{(p-q+\hat{\delta})^{2}-4+\hat{\epsilon}^{2}}\right]+\frac{\pi}{2} \operatorname{Sign}(\hat{\epsilon}) H\left(4-(p-q+\hat{\delta})^{2}-\hat{\epsilon}^{2}\right),
\end{aligned}
$$

where $\hat{\delta} \equiv \delta-\delta^{\prime}, \hat{\epsilon} \equiv \epsilon-\epsilon^{\prime}$, and $H(x)$ is the Heaviside step function, Clearly, in the limit $L \rightarrow \infty$, equivalently $\hat{\epsilon}, \hat{\delta} \rightarrow 0$, we have $\theta\left(\lambda_{1}, \lambda_{2}\right) \rightarrow \pm i \infty$ for $p-q=\mp 2$. These divergences are reflected in exponentially vanishing or diverging amplitudes in (7). The treatment of these singularities in constructing the eigenstates of the Heisenberg chain can be tricky and it is detailed in Appendix B.1, Appendix B.2, and Appendix C for the string configurations considered in this work.

\section{Few-particle entanglement: Eigenstates with $M=2$ particles}

In this section, as an extreme case of the vanishing density limit (i.e., with fixed $M$ and $L \rightarrow \infty)$, we discuss analytical results for the entanglement entropy and entanglement spectrum (ES) of the eigenstates of the $X X X$ chain in the sector with two particles 
$M=2$. We consider both 1-string and 2-string eigenstates (see sections 2.2 and 2.3). The former are states of two unbound particles (magnons), whereas the latter correspond to bound states of the two particles. The analytical results obtained from the Bethe ansatz solution are discussed in Appendix A and Appendix B.1.

Based on the analytical solution, we present a semiclassical upper bound $S_{M A X}(M=2$ for the von Neumann entropy (see Appendix A and Appendix D). In the 1-string sector this is saturated in the limit $L \gg 1$. On the other hand, for 2-string eigenstates the entanglement entropy is dramatically reduced, and we find $S_{M A X}(M=1)<S_{A}<$ $S_{M A X}(M=2)$.

We also examine the dependence of the entanglement entropy on the eigenstate energy, focusing on the half-chain entropy $S_{A}(L / 2)$. In the 1-string sector this exhibits a twoband structure. In both bands the entropy is approximately constant as a function of energy, and in the upper one $S_{A}(L / 2) \approx S_{M A X}(M=2, L / 2)$. These bands can be understood in terms of the two Bethe-Takahashi quantum numbers used to identify the eigenstates of the $X X X$ chain (see section 2). Precisely, the entanglement entropy increases with the number of blocks of contiguous Bethe quantum numbers: while the lower band correspond to two quantum numbers next to each other (i.e., a single block), the upper one is obtained for two quantum numbers far apart (two blocks). Equivalently, the first situation correspond to the two particles having similar quasi-momenta, while the latter is reflected in particles with very different ones.

In the 2-string sector the entanglement entropy changes continuously (in the large $L$ limit) with the eigenstate energy, and no entanglement bands are observed. This reflects the behavior of the bound-state length $\ell_{b}$ (cf. (26)), which is a continuous increasing function of the bound-state energy (cf. (27)). The entanglement entropy generically increases upon increasing $\ell_{b} / L$. For $\ell_{b} / L \rightarrow 0$ (i.e. two strongly-bound particles) one has $S_{A}(L / 2) \approx S_{M A X}(M=1, L / 2)$.

All these findings are reflected at the level of the half-chain entanglement spectrum. In the 1-string sector the ES levels obtained from highly-entangled eigenstates (i.e., saturating the upper bound $S_{M A X}$ ) are simple functions of the ratio $\omega \equiv L_{A} / L$ that can be understood semiclassically (see Appendix A for analytical results). Surprisingly, for 2-string eigenstates, besides the usual bulk ES levels, edge-related ES levels appear, which diverge in the limit $L \gg 1$. The corresponding entanglement eigenfunctions are exponentially localized at the boundary between the two subsystems (edge-states).

\subsection{Overview: interacting magnons versus bound states}

The entanglement entropy $S_{A}\left(L_{A}\right)$ obtained from the eigenstates of the Heisenberg chain in the sector with $M=2$ particles is plotted versus the block length $L_{A}$ in Figure 1. Symbols are exact numerical data for $S_{A}$ obtained from the Bethe ansatz result (6), for a chain with $L=32$. All the possible eigenstates are considered in the figure. Panels (a) and (b) show the entanglement entropy for 1-string and 2-string eigenstates, respectively. 

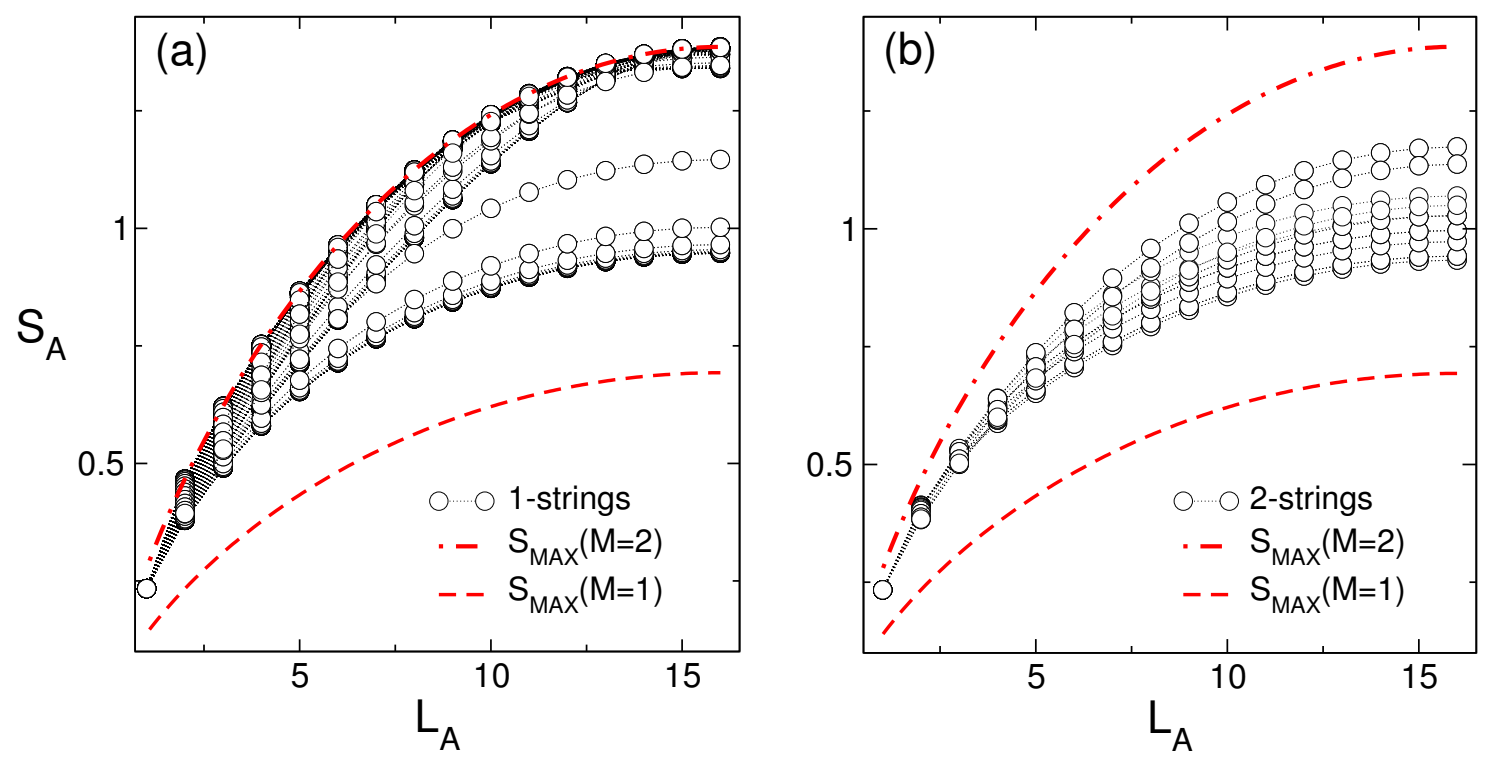

Figure 1. Entanglement of the eigenstates of the Heisenberg spin chain in the sector with two particles $(M=2)$ : entanglement entropy $S_{A}$ versus the subsystem size $L_{A}$ (dotted lines are guides to the eye). Data are for a chain of length $L=32$. (a) Eigenstates corresponding to real solutions of the Bethe equations (1-strings). The dashed and dash-dotted lines the (lower and upper) bounds $S_{M A X}(M=1)$ and $S_{M A X}(M=2)$, respectively, with $S_{M A X}(M)=-M(\omega \log (\omega)+(1-\omega) \log (1-\omega))$ and $\omega \equiv L_{A} / L$ (b) Eigenstates corresponding to pairs of complex conjugate solutions of the Bethe-Takahashi equations (2-strings): $S_{A}$ versus $L_{A}$ (same scale as in (a) on the $y$-axis). Dashed and dash-dotted lines are the same as in (a). Note the lower value of entanglement and the dense structure as compared to the case of 1-strings in (a).

We start discussing the 1-strings. The semiclassical upper-bound (5) for the von Neumann entropy is reported in Figure 1 (a) for $M=1$ and $M=2$ as dashed and dashdotted line, respectively. Clearly, we have $S_{M A X}(M=1)<S_{A}\left(L_{A}\right) \lesssim S_{M A X}(M=2)$, i.e., the upper bound is almost saturated. Deviations from $S_{M A X}(M=2)$ in the upper band are $\mathcal{O}(1 / L)$.

The entanglement entropy obtained from 2-string eigenstates is shown in Figure 1 (b). Dashed and dash-dotted lines are the same as in panel (a). A dramatic reduction in the entanglement entropy is observed, as compared with the upper-band of the 1-string eigenstates (panel (a)). Interestingly, we have $S_{A}\left(L_{A}\right)>S_{M A X}(M=1)$, contradicting the naive picture of the bound state as an effective single particle. This reflects the effective bound-state length $\ell_{b}$ (cf. (26)) being finite. Only for low-energy 2-strings one has $\ell_{b} / L \rightarrow 0$ and $S_{A}\left(L_{A}\right) \approx S_{M A X}(M=1)$ in the limit $L \gg 1$.

\subsection{Entanglement versus energy: entanglement bands}

The behavior of the half-chain entanglement entropy $S_{A}(L / 2)$ as a function of the eigenstate energy $E$ is illustrated in Figure 2. Data are the same as in Figure 1. Panel (a) and (b) are for the 1-string and the 2-string eigenstates, respectively. Notice that 


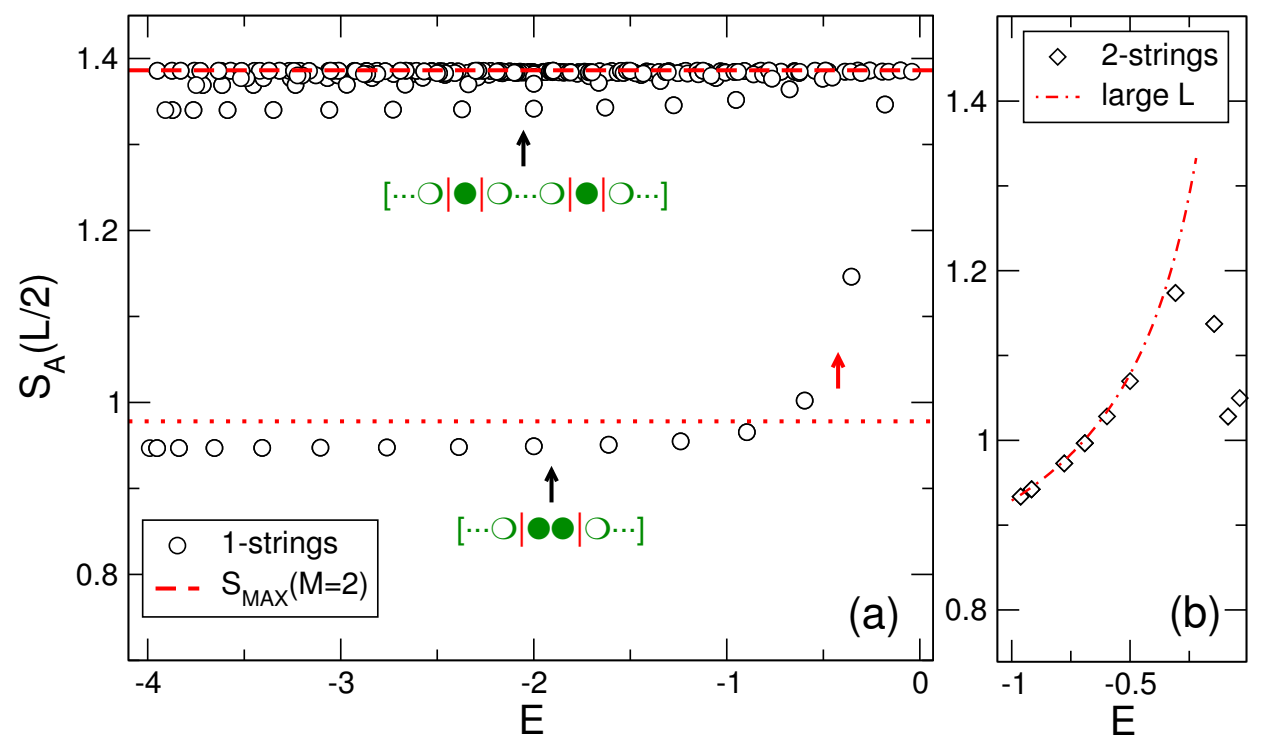

Figure 2. Eigenstates of the Heisenberg spin chain in the sector with two particles $(M=2)$ : entanglement bands. Half-chain entanglement entropy $S_{A}(L / 2)$ versus eigenstate energy $E$. Data are Bethe ansatz results for a chain with $L=32$. (a) 1-string eigenstates. The dashed line is $S_{M A X}(M=2)$ for $\left.L_{A}=L / 2\right)$. Two bands with approximately constant entropy (as a function of energy) are visible. The cartoons show typical Bethe-Takahashi number configurations for the eigenstates in the bands. Empty (full) circles denote vacant (occupied) quantum numbers. The vertical lines mark disconnected blocks of occupied quantum numbers. The lower and the upper entanglement band corresponds to configurations with two neighboring and two separated Bethe quantum numbers, respectively. The vertical arrow is to stress the merging of the two bands at high energy. (b) 2-string eigenstates. No band structure is visible. The dash-dotted line is the analytical result in the large chain limit $L \gg \ell_{b}$, with $\ell_{b}$ the size of the bound state.

while for the 1-strings we have $-4<E<0,2$-strings correspond to the higher energies $-1<E<0$ (cf. (25)).

In the 1-string sector $S_{A}(L / 2)$ exhibits a two-band structure. The lower band with $S_{A}(L / 2) \approx 1$ corresponds to eigenstates obtained from two Bethe-Takahashi numbers (see section 2) $\left\{I_{1}^{(1)}, I_{2}^{(1)}\right\}$ next to each other (i.e. $\delta I \equiv\left|I_{2}^{(1)}-I_{1}^{(1)}\right|=1$ ). This is illustrated by the cartoon in the Figure, where full (empty) circles denote occupied (vacant) Bethe quantum numbers (see section 2.3), whereas vertical lines mark the disconnected blocks of occupied quantum numbers. Since $\delta I=1$ implies $\left|k_{2}-k_{1}\right| \sim 2 \pi / L$ (at least in the $L \gg 1$ limit, cf. Appendix A.1), the lower band corresponds to eigenstates with particles with similar quasi-momenta. In particular, the dotted line in the Figure is the analytical result (see Appendix A.1) assuming $\left|k_{2}-k_{1}\right|=2 \pi / L$, and it is in agreement with the data, apart from $\mathcal{O}(1 / L)$ corrections.

The upper band corresponds to $\left|I_{2}^{(1)}-I_{1}^{(1)}\right|>1$, i.e., eigenstates obtained from two separated Bethe quantum numbers (as shown by the cartoon). The substructures (lines) within the band correspond to shifting two equi-distant (i.e., with $\delta I=$ const.) quantum 
numbers.

These substructure are generic for eigenstates with finite $M$ and $L \rightarrow \infty$. Precisely, we observe that a global shift of the Bethe-Takahashi numbers, which amounts to a shift in the eigenstate total quasi-momentum (cf. (13)), does not change significantly the entanglement entropy. This is better understood in the non-interacting limit (i.e., the $X X$ chain), where $k_{\alpha} \sim 2 \pi J_{\alpha} / L$ [121], implying that a global shift of the Bethe numbers corresponds to shifting the quasi-momenta as $k_{\alpha} \rightarrow k_{\alpha}+$ $\delta k$. As a consequence, the wavefunction amplitude $A_{M}\left(x_{1}, x_{2}, \ldots, x_{M}\right)$ (cf. (7)) is modified as $A_{M}\left(x_{1}, x_{2}, \ldots, x_{M}\right) \rightarrow e^{i \delta k \sum_{i} x_{i}} A\left(x_{1}, x_{2}, \ldots, x_{M}\right)$. Since the phase factor $\exp \left(i \delta k \sum_{i} x_{i}\right)=\exp \left(i \delta k \sum_{j \in A} x_{j}\right) \times \exp \left(i \delta k \sum_{j \in B} x_{j}\right)$ corresponds to a product of unitary transformations on the subsystems Hilbert spaces $\mathcal{H}_{A}$ and $\mathcal{H}_{B}$, it does not affect the entanglement spectrum and entropy. This observation can be recast in the CFT language, which allows to generalize it to other interacting $c=1$ and for finite particle density $M / L$. In fact, the total quasi-momentum shift is implemented in the CFT language by acting over the ground state with vertex operators $Y_{1}\left[\alpha_{+}, \alpha_{-}\right]=e^{i\left(\alpha_{+} \phi+\alpha_{-} \bar{\phi}\right)}$, where $\phi$ and $\bar{\phi}$ are bosonic operators, and $\alpha_{ \pm}$are related to their scaling dimensions. Remarkably, it has been shown in Ref. [71] that all the excitations obtained in this way have the same entropy as the ground state.

Interestingly, the results in Figure 2 suggest that, at least in the vanishing density regime, the effect of the scattering phases is negligible at low energy. This does not hold at high energies $\left(E \rightarrow 0^{-}\right.$) where the two bands merge. This corresponds to $I_{1}^{(1)} \approx I_{2}^{(1)} \approx I_{M A X}^{(1)}\left(\right.$ cf. (21)), which implies $k_{1} \approx k_{2} \approx 0 \bmod 2 \pi$ (cf. (A.8)). Notice that for $k_{1}, k_{2} \rightarrow 0$, since the wavefunction (6) becomes "flat", the semiclassical bound $S_{M A X}$ is exact (see Appendix D).

The entanglement entropy for the bound states of two particles is plotted in Figure 2 (b). In contrast with the 1-strings (panel (a)), no band structure is visible. The entanglement entropy increases monotonically up to $E \approx-0.3$, when it starts decreasing. Interestingly, one has $S_{A}(L / 2)>S_{M A X}(M=1, L / 2)=\log (2)$, which would be the entropy of a one particle state. This behavior can be understood in terms of the bound-state length $\ell_{b}$ (cf. (27)). At $E=-1$ we have $\ell_{b}=0$, implying $S_{A}(L / 2) \approx \log (2)$. On the other hand, as $E \rightarrow 0^{-}, \ell_{b} \rightarrow \infty$ and the two particles become weakly bound, which is associated with a substantial increase in the entanglement. More quantitatively, for $\ell_{b} \lesssim L$ one has (see Appendix B.1 for the derivation)

$$
S_{A}(L / 2)=\log (2)-\frac{2}{L E}[1-\log (2)+\log (L)+\log (-E)]+\mathcal{O}\left(1 / L^{2}\right)
$$

which is shown as dash-dotted line in the Figure. Notice that (30) breaks down at

$E \approx-0.3$. This can be understood considering the entanglement spectrum (ES) of 2-string eigenstates.

\subsection{Entanglement spectra}

In the 1-string sector, the half-chain ES contains three levels $\xi_{0}$ and $\xi_{ \pm}$, which are functions of the relative quasi-momentum $k \equiv k_{2}-k_{1}$ between the two particles 

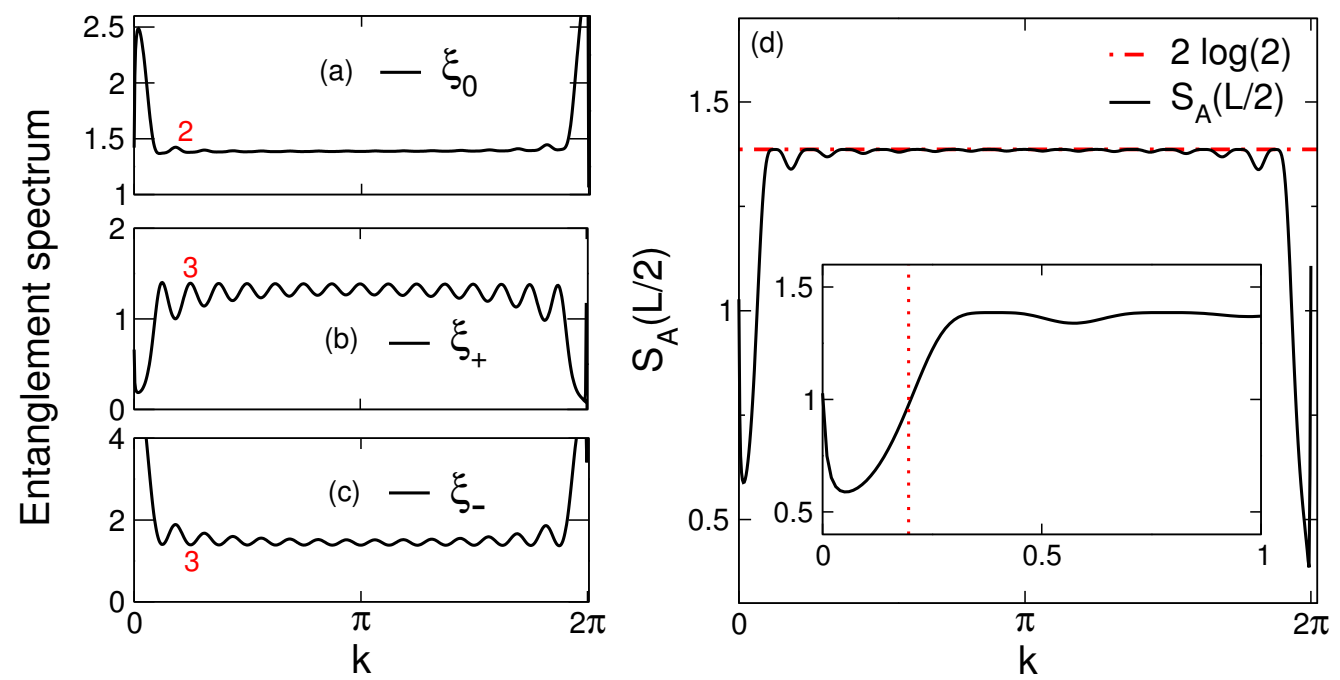

Figure 3. Entanglement spectrum (ES) and entropy for the Heisenberg chain in the sector with two particles $M=2$. (a)(b)(c) ES levels $\xi_{+}, \xi_{-}, \xi_{0}$ plotted versus the relative momentum $k \equiv k_{2}-k_{1}$ of the two particles. Data are exact results for a chain with $L=32$. For $0 \ll k \ll 2 \pi$ we have $\xi_{0} \approx \xi_{+} \approx \xi_{-} \approx 2 \log (2)$. The accompanying numbers specify the degeneracies of the levels. The resulting half-chain entropy $S_{A}$ is reported in panel (d) versus $k$ (full line). The dash-dotted line corresponds to the upper bound $S_{M A X}=2 \log (2)$. Inset: same as in the main figure zooming around $k \approx 0$. The vertical dotted line is $k=2 \pi / L$.

(see Appendix A). These are shown in Figure 3 (panels (a)-(c)) plotted versus $k$, for a chain with $L=32$. The accompanying numbers in the panels specify the degeneracies of the ES levels. To be general we keep $k$ arbitrary, while in principle its value is fixed by using the solutions of the Bethe equations (12). Moreover, data in Figure 3 are for fixed relative scattering phase (cf. (8)) $\theta \equiv \theta_{2,1}-\theta_{1,2}=\pi$. We verified numerically that the main results do not change for different values of $\theta$, at least for $0 \ll k \ll 2 \pi$ (central region in the panels), where the semiclassical approximation holds (see Appendix A). As Figure 3 shows, for $0 \ll k \ll 2 \pi$ one has $\xi_{0} \approx \xi_{+} \approx \xi_{-} \approx 2 \log (2)$. These results are correct up to terms $\mathcal{O}(1 / L)$, and can be derived rigorously using the Bethe ansatz solution (see Appendix A). This implies, as expected, that in the semiclassical regime the effect of the scattering phase $\theta$ (cf. (8)) can be neglected. This scenario breaks down at $k \sim 0 \bmod 2 \pi$, where deviations from the semiclassical results are observed for all ES levels (cf. Figure 3). The half-chain entanglement entropy obtained from the ES in (a)-(c) is reported in Figure 3 (d) as a function of $k$. While at $k \neq 0$ the entanglement entropy exhibits a flat behavior with $S_{A}(L / 2) \approx S_{M A X}(M=2)=2 \log (2)$ (dash-dotted line in the Figure), at $k \rightarrow 0 \bmod 2 \pi$ a strong reduction occurs. This is better highlighted in the inset of the Figure zooming in the region $0 \leq k \leq 1$. The vertical dotted line is $k=2 \pi / L$, the value $S_{A}(L / 2) \approx 1$ is the horizontal dotted line shown in Figure 2.

We now turn to the entanglement spectra of 2-string eigenstates. The calculation of the ES is outlined in Appendix B.1. The ES contains four levels, which are plotted 

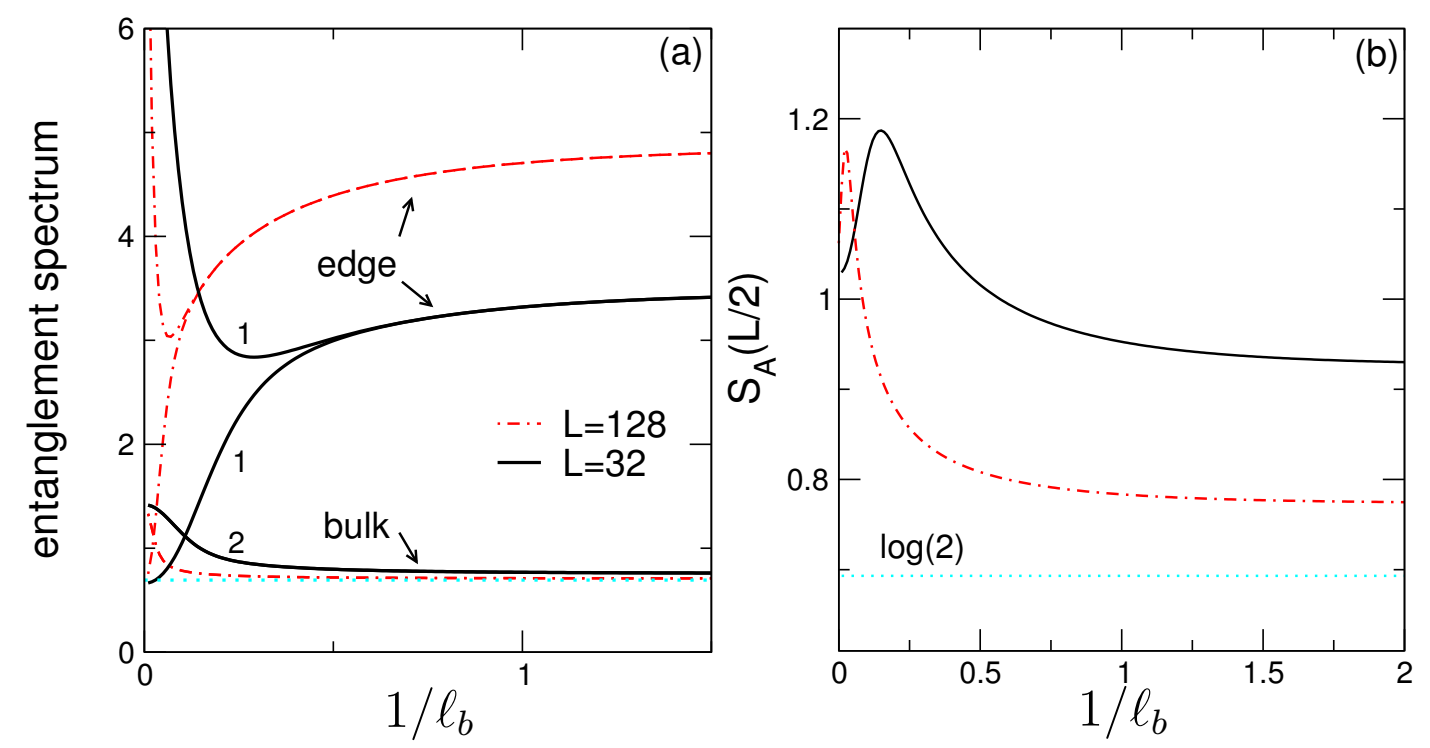

Figure 4. Entanglement spectrum and entanglement entropy for 2-string eigenstates of the $X X X$ chain (bound states of two particles). (a) Entanglement spectrum levels $\xi$ plotted versus $1 / \ell_{b}, \ell_{b}$ being the bound-state length. Full and dash-dotted line are exact numerical results for a chain with $L=32$ and $L=128$, respectively. Accompanying numbers specify the degeneracies (shown only for $L=32$ ) of the levels. Two edgerelated ES levels are present, besides the bulk ones (with $\xi \approx \log (2)$ at $\ell_{b} \rightarrow 0$ ). At $\ell_{b} \rightarrow \infty$ one of the two edge levels diverges, whereas the remaining is $\sim \log (2)$, i.e., an extra bulk ES level appears. (b) Half-chain entanglement entropy $S_{A}(L / 2)$ obtained from the ES levels in panel (a) plotted versus $1 / \ell_{b}$. Notice that $S_{A}(L / 2) \rightarrow \log (2)$ at $\ell_{b} \rightarrow 0 L \rightarrow \infty$, while $S_{A}(L / 2) \rightarrow 3 / 2 \log (2)$ for $\ell_{b} \rightarrow 0$.

in Figure 4 versus the inverse bound-state length $1 / \ell_{b}$ (cf. (26) and (27)). The full and dash-dotted lines are for chains of length $L=32$ and $L=128$, respectively. The accompanying numbers specify the degeneracies of the ES levels. Clearly, two of the levels are $\sim \log (2)$ as $1 / \ell_{b} \rightarrow 0$ (horizontal line in the Figure), i.e., for eigenstates containing two strongly-bound particles. These are "bulk" ES levels, similar to the ES levels for two unbound particles (cf. Figure 3). Notice that, at fixed $\ell_{b}$ deviations from $\log (2)$ are $\mathcal{O}(1 / L)$, as can be confirmed by comparing the curves for $L=32$ and $L=128$. Strikingly, the remaining two ES levels diverge in the limit $\ell_{b}, L \rightarrow \infty$. These are edge-related levels. The corresponding entanglement eigenvectors (i.e., eigenvectors of the reduced density matrix) are exponentially localized at the two edges of subsystem $A$ (edge states) (cf. Appendix B.1). Interestingly, at $1 / \ell_{b} \approx 1 / 2$ one of the two edge levels diverges, whereas the other one becomes an extra "bulk" level. This corresponds to the maximum entropy in Figure 2 (b), where (30) breaks down. The half-chain entanglement entropy obtained from the ES in panel (a) is shown in Figure 3 (b) as a function of $1 / \ell_{b}$. In the limit $1 \ll \ell_{b} \ll L$ we have $S_{A}(L / 2)=\log (2)$, i.e. the half-chain entropy of a single particle. Notice that for $\ell_{b} \rightarrow \infty$ we have $S_{A}(L / 2) \approx 3 / 2 \log (2)$, due to the extra bulk level appearing in the ES (see panel (a)). 

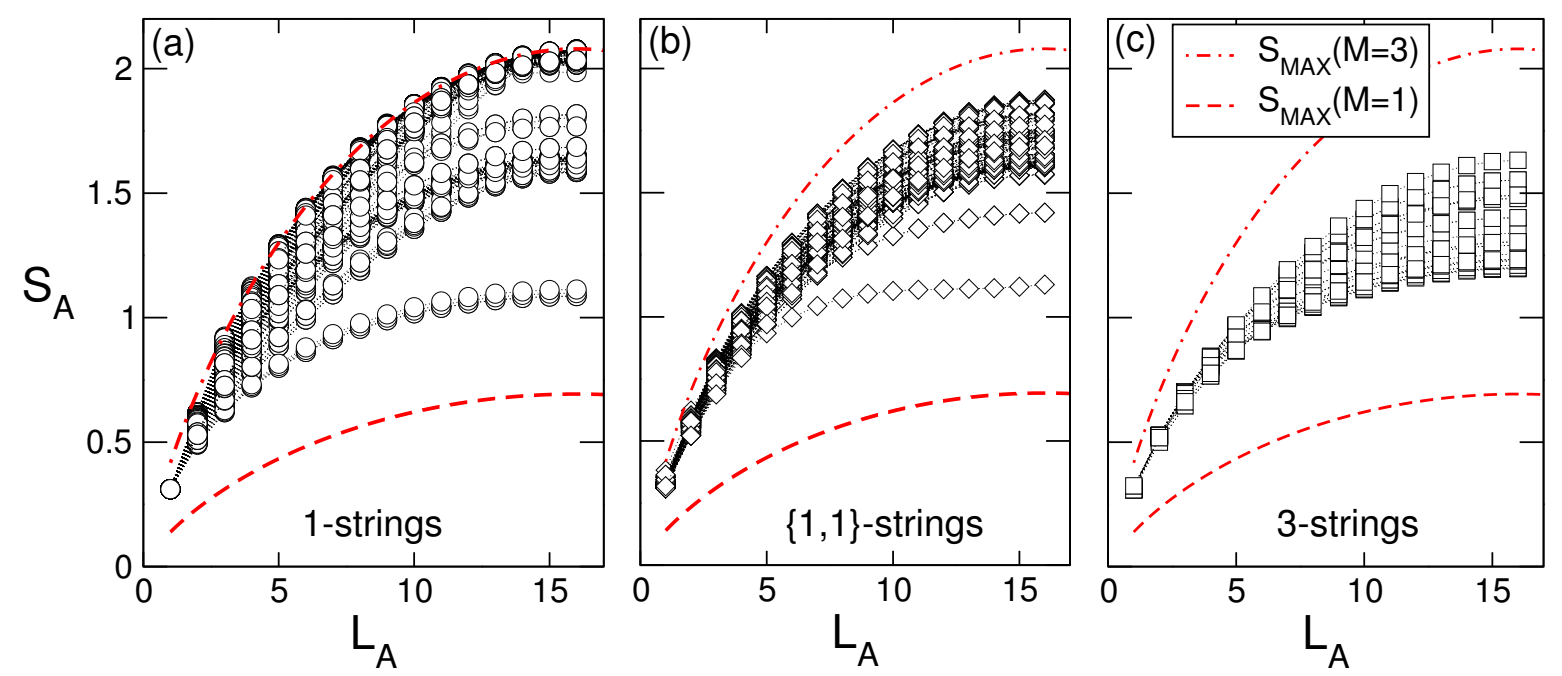

Figure 5. Entanglement entropy of the eigenstates of the $X X X$ chain in the sector with three particles $M=3$. Data are exact numerical data for $L=32$ sites. (a) 1-string eigenstates: $S_{A}$ plotted versus the size of block $A L_{A}$. The dashed line is the upper bound $S_{\text {MAX }}(M=3) \equiv 3 \log (2)$. The dash-dotted line is $S_{M A X}(M=1) \equiv 2 \log (2)$ (cf. Figure 1). (b) Eigenstates corresponding to $\{1,1\}$-strings, i.e. one real and a pair of complex conjugate solutions of the Bethe equations. (c) Same as in (a)(b) but for 3 -string eigenstates (i.e., bound states of the three particles).

\section{Few-particle entanglement : Eigenstates with $M=3$ particles}

In this section we focus on entanglement properties in eigenstates of the $X X X$ chain in the sector with $M=3$. Besides 1-string and 3-string eigenstates, which correspond to unbound particles and three-particle bound states, the intermediate situation with eigenstates that contain a magnon and a two-particle bound state arises. These correspond to solutions of the Bethe-Takahashi equations (20) with string content $\{1,1\}$ $(\{1,1\}$-strings), i.e. one real and two complex conjugate rapidities.

For highly-entangled 1-string eigenstates we show that $S_{A}\left(L_{A}\right) \lesssim S_{M A X}(M=3)$, confirming what has been found for two particles in section 3. Furthermore, the entanglement entropy is progressively reduced in eigenstates that contain bound states (i.e. $\{1,1\}$-strings and 3-strings). We also investigate the behavior of the half-chain entanglement entropy as a function of the eigenstate energy. For the 1-strings we observe an entanglement band-like structure with three bands. As in the two-particle case (see section 3)), higher entanglement bands corresponds to a larger number of blocks of contiguous Bethe-Takahashi quantum numbers. In the sector with $\{1,1\}$-strings, the half-chain entropy exhibits a dense structure (i.e., no entanglement bands), reflecting that the size $\ell_{b}$ of the two-particle bound state varies continuously as a function of the bound-state energy. 


\subsection{Overview: unbound particles, bound states, and coexistence of the two behaviors}

The von Neumann entropy $S_{A}\left(L_{A}\right)$ for the eigenstates of the $X X X$ chain in the sector with $M=3$ is shown in Figure 5 (panels (a)-(c)) for a chain with $L=32\left(S_{A}\right.$ plotted versus the subsystem size $L_{A}$ ). The three panels (a)-(c) correspond to eigenstates obtained from different types of solutions of the Bethe-Takahashi equations: 1-strings, $\{1,1\}$-strings, and 3-strings are shown in panels (a),(b), and (c). The same scale is used on the $y$-axis in all the panels. The dashed and dash-dotted lines denote the semiclassical bound $S_{M A X}(M)$ (cf. (5)) with $M=2$ and $M=3$, respectively. The construction of the Bethe wavefunctions, from which the results in the Figure are derived, is outlined in Appendix C and Appendix B.2.

Clearly, $S_{M A X}(M=3)$ is saturated (apart from $\mathcal{O}(1 / L)$ terms) in the highest band of the 1-string sector. A substantial decrease in the entanglement entropy occurs for $\{1,1\}$-strings. Large values of $S_{A}\left(L_{A}\right)$ correspond to eigenstates of the $X X X$ chain that contain an unbound particle and a pair of weakly bound particles, whereas small ones are obtained when two strongly-bound particles are present. Finally, a further reduction of the entanglement entropy happens for eigenstates obtained from 3-strings (panel (c)). However, $S_{A}\left(L_{A}\right)>S_{M A X}(M=1)$, reflecting that the size $\ell_{b}$ of the three-particle bound is finite, similarly to the two-particle bound states (see Figure 1).

\subsection{Entanglement entropy versus energy: bands and continuous structures}

The half-chain entanglement entropy $S_{A}(L / 2)$ is plotted versus the eigenstate energy in Figure 6 for 1 -strings, $\{1,1\}$-strings, and 3-strings. Data are the same as in Figure 5 (in all the panels the same scale on the $y$-axis is used). Notice that 1-string eigenstates have energies $-6 \lesssim E \lesssim 0$, while $\{1,1\}$-strings and 3 -strings appear at $-3 \lesssim E \lesssim 0$, and $-2 / 3 \lesssim E \lesssim 0$, respectively.

In the 1-string sector (panel (a) in the Figure) $S_{A}(L / 2)$ exhibits three bands, which correspond to eigenstates obtained from Bethe-Takahashi quantum numbers with different numbers of blocks of contiguous quantum numbers (as shown in the cartoons, see also section 2). $S_{A}(L / 2)$ increases as a function of the number of Bethe number blocks (from one for the lowest band up to three for the highest one). Moreover, $S_{A}(L / 2) \approx S_{M A X}(M=3)$ (dashed line in panel (a)) for the eigenstates with three separated Bethe numbers. At high energy $E \rightarrow 0^{-}$all the bands tend to merge, as it has been observed for two particles (see Figure 2).

The half-chain entropy for $\{1,1\}$-string eigenstates is shown in panel (b). A denser (as compared with 1-strings) structure appears, reflecting that the length $\ell_{b}$, which is associated with the two-particle bound state, is a continuous function of the energy.

Finally, $S_{A}(L / 2)$ for 3 -string eigenstates is shown in panel (c). The dash-dotted line in the Figure is the analytical result in the limit $L \gg \ell_{b}$ (cf. (B.24) and (B.25)),

$$
S_{A}(L / 2)=\log (2)-\frac{6}{2 L E}[1+2 \log (2)+\log (L)+\log (-E)-\log (6)]+\mathcal{O}\left(1 / L^{2}\right),
$$

which accurately describes the behavior of the entropy in the region $E \lesssim-0.3$. Notice 

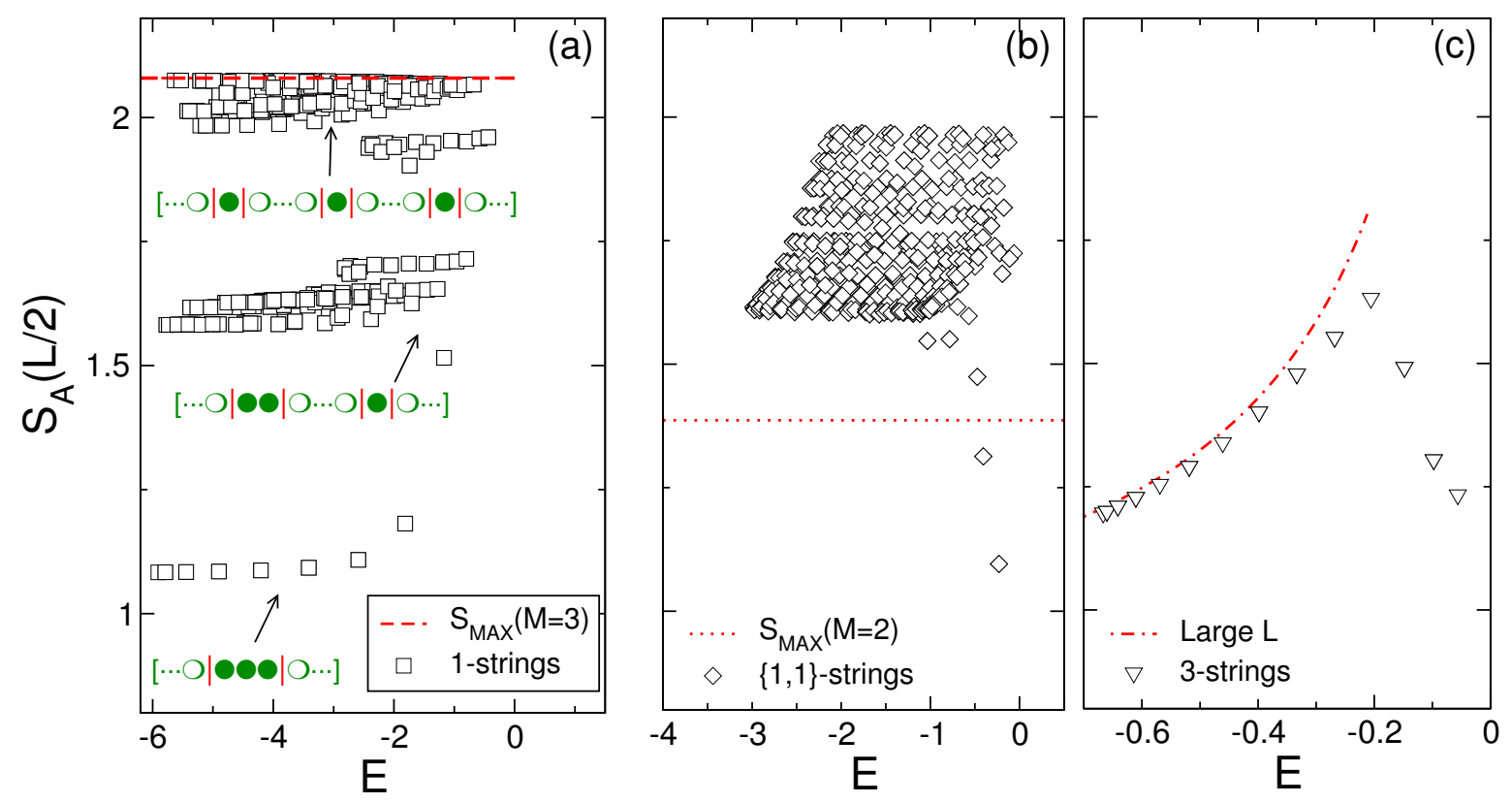

Figure 6. Entanglement entropy for the Heisenberg chain in the sector with three particles $M=3$. Half-chain entropy $S_{A}(L / 2)$ plotted versus the eigenstate energy $E$. Data are exact numerical result from the Bethe ansatz solution of the model for a chain with $L=32$ sites. (a) 1 -string eigenstates: three entanglement bands are visible. The cartoons show typical Bethe-Takahashi number configurations corresponding to eigenstates in a given band. Full and empty symbols denote occupied and vacant Bethe quantum numbers, respectively. The vertical lines mark disconnected blocks of occupied numbers. Different points in a given band are obtained by shifting the blocks independently. The dashed line is the upper bound $S_{M A X}(M=3)=3 \log (2)$. (b) The same as in (a) for $\{1,1\}$-string eigenstates. (c) Same as in (a)(b) for the three-particle bound states (3-strings). The dash-dotted line is the analytical result in the large $L$ limit.

that deviations from $S_{A}(L / 2) \sim \log (2)$ are $\propto 1 /(L E)$, as for the two-particle bound state (cf. (30)). Interestingly, $S_{A}(L / 2)$ exhibits a maximum with $S_{A}(L / 2) \approx 1.75$ at $E \approx-0.2$, similar to the case of a two-particle bound state (see Figure 2 and 3 ).

All these findings can be also understood at the level of the half-chain entanglement spectrum. In particular, the ES of 1-string eigenstates with maximum number of blocks (three separated Bethe numbers) exhibits $2^{M}=8$ degenerate levels $\xi=\log (8)$, in agreement with the semiclassical result in Appendix D. On the other hand, the ES of the three-particle bound states is qualitatively similar to what has been found for the two-particles (see Appendix B.2 for some analytical results). It is interesting, however, to consider the entanglement spectrum of $\{1,1\}$-strings.

\subsection{Entanglement spectrum of $\{1,1\}$-string eigenstates}

The ES for eigenstates of the $X X X$ chain obtained from $\{1,1\}$-strings is shown in Figure 7. Data are obtained from the Bethe ansatz wavefunction (6) for a chain with 

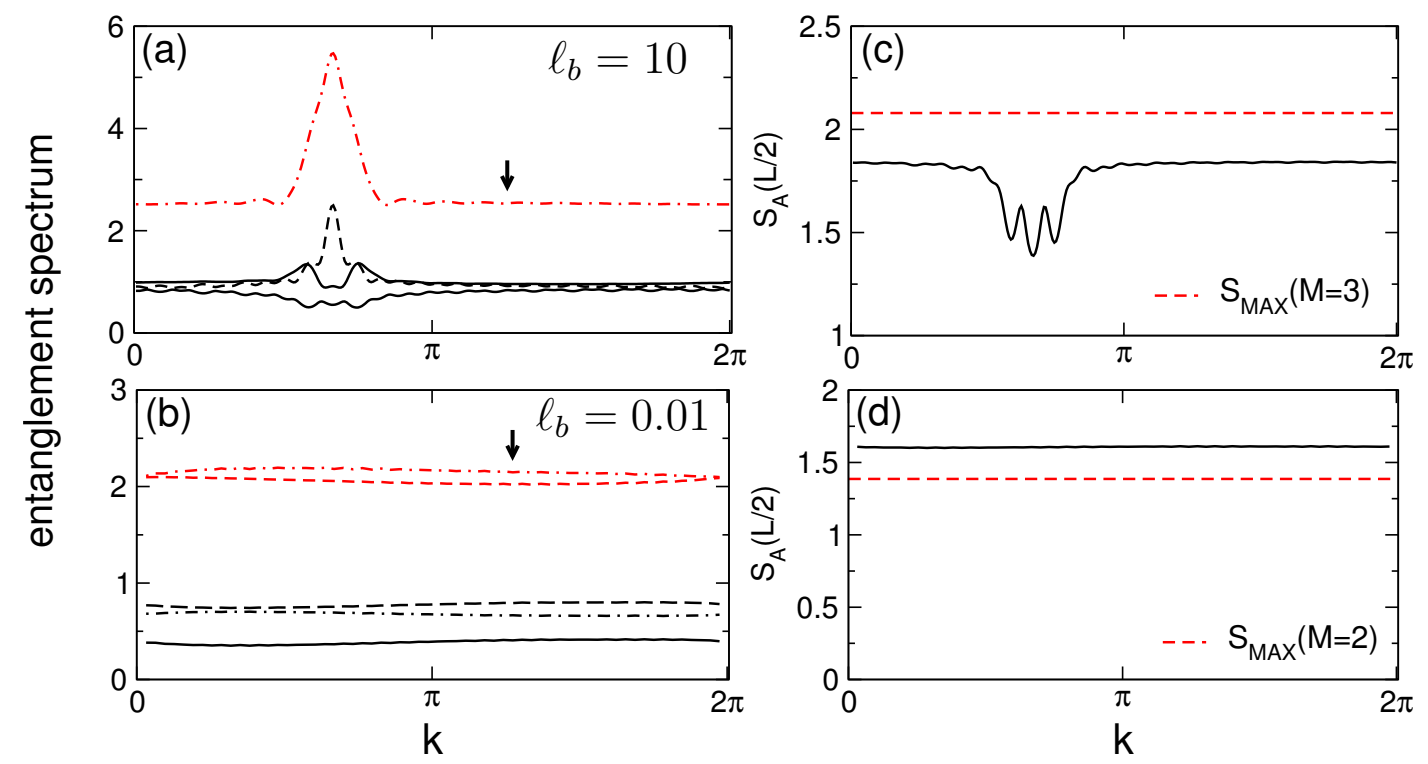

Figure 7. Entanglement entropy and entanglement spectrum (ES) of the Heisenberg spin chain in the sector with $M=3$ particles. Only $\{1,1\}$-string eigenstates are shown in the figure. These comprise a "free" particle and a two-particle bound state. Data are exact results from the Bethe ansatz solution for a chain with $L=32$ sites. (a)(b) Half-chain ES for a fixed momentum of the bound state $K_{b}=2 \pi / 3$ plotted versus the "free" particle quasi-momentum $k$. Panels (a) and (b) are for different values of the bound state length $\ell_{b}=10$ (two weakly-bound particles) and $\ell_{b}=0.01$ (two stronglybound particles). The arrow marks edge-related ES levels. (c) Half-chain entropy $S_{A}(L / 2)$ obtained from the ES in panel (a). The dashed line is the semiclassical upper bound $S_{\text {MAX }}(M=3)$ (cf. (5)). Same as in (c) for the ES in panel (b). The dashed line is now $S_{M A X}(M=2)$.

$L=32$ sites. For simplicity we fix in (6) the total bound state quasi-momentum to $K_{b}=2 \pi / 3$, leaving as free parameter the quasi-momentum $k$ of the "free" particle. Moreover, we treat the bound state length $\ell_{b}$ as an independent variable, although it can be related to the total bound-state quasi-momentum $K_{b}$ via (26) and (27). The construction of the Bethe wavefunction for $\{1,1\}$-strings is outlined in Appendix C.

Panels (a) and (b) in Figure 7 show the half-chain ES levels as a function of $k$ for $\ell_{b}=10$ (two weakly-bound particles) and $\ell_{b}=10^{-2}$ (two strongly-bound particles), respectively. The corresponding entanglement entropies are reported in panels (c) and (d). In the case of two weakly-bound particles (panel (a)) the ES exhibits four levels. In the limit $L \rightarrow \infty$ three ES levels are $\sim \log (2)$ (dotted line), whereas the remaining one is diverging as $\sim \log (L)$. This is the same edge level found in the ES of the two-particle bound states in section 3 (see Figure 4). Notice that the ES changes dramatically at $k \approx K_{b}=2 \pi / 3$, which is reflected in a reduction in the entanglement entropy (see panel (c)). For two strongly-bound particles (see panel (b)) two edge levels appear (as for two particles, see Figure 4). Interestingly, the ES does not exhibit any significant dependence on $k$, and one has $S_{A}(L / 2) \approx S_{M A X}(M=2) \forall k$. 

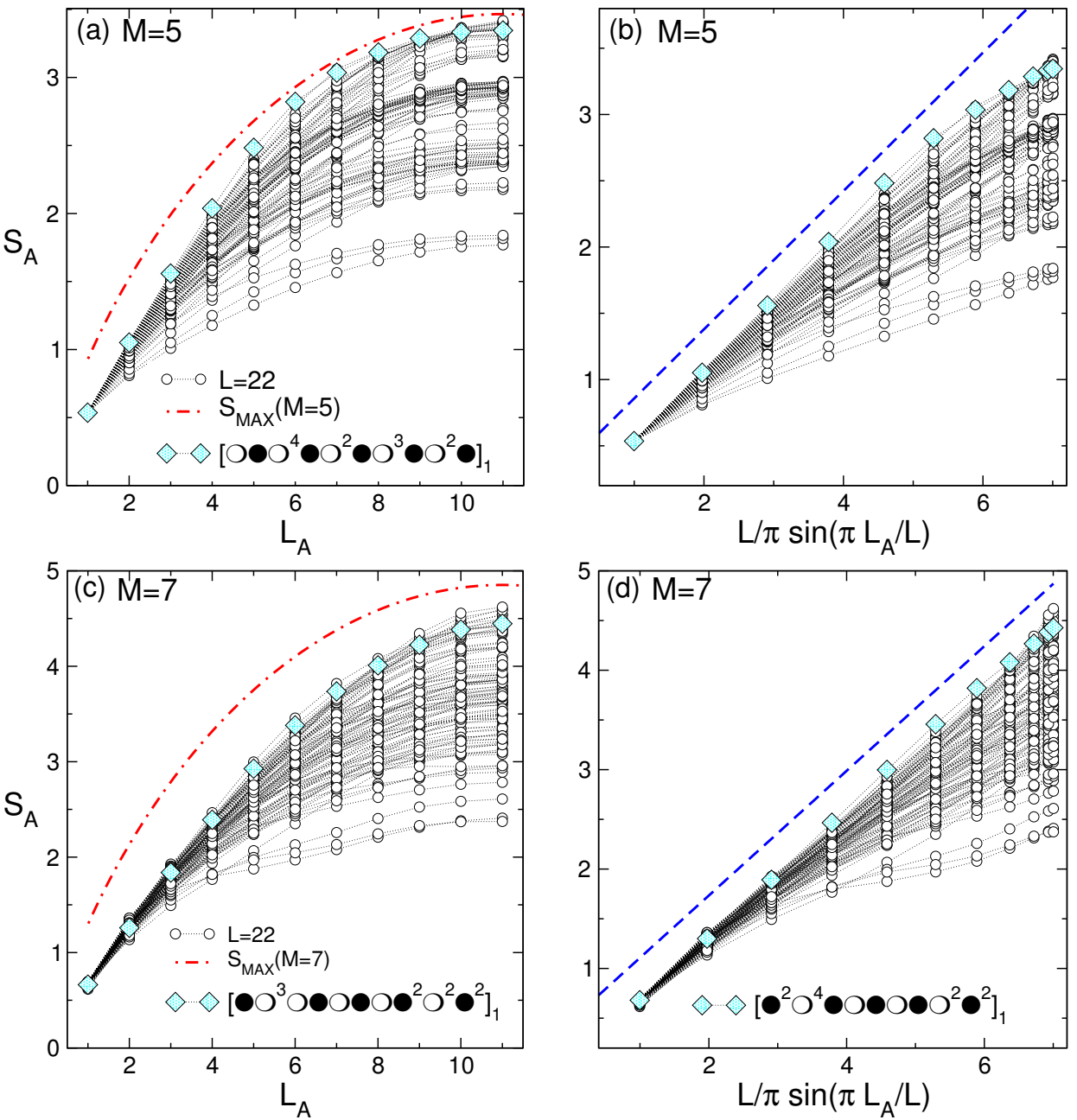

Figure 8. Volume law (i.e. extensive behavior) in the entanglement entropy of the eigenstates of the $X X X$ chain. Here we focus on 1-string eigenstates. Data are exact numerical results obtained from the Bethe ansatz solution of the model for a chain with $L=22$ sites. (a) Sector with $M=5$ : entanglement entropy $S_{A}$ plotted versus the block length $L_{A}$. The dash-dotted line is the upper bound $S_{M A X}(M)=-M[\omega \log (\omega)+(1-\omega) \log (1-\omega)]$, with $\omega \equiv L_{A} / L$. (b) Same data as in (a) plotted versus the chord length $L_{c} \equiv(L / \pi) \sin \left(\pi L_{A} / L\right)$. The dash-dotted line is a guide to the eye. Full symbols (rhombi) denote $S_{A}$ obtained from the eigenstate with Bethe-Takahashi quantum numbers $\left[\circ \circ^{4} \bullet \bigcirc^{2} \bullet \bigcirc^{2} \bullet \bigcirc^{2} \bullet\right]_{1}$, where $\bullet$ and $\bigcirc$ denote occupied and vacant quantum numbers, respectively. (c)(d) The same as in panels (a)(b) now in the sector with $M=7$.

\section{Entanglement of eigenstates with a finite density of particles}

In this section we focus on the behavior of the entanglement entropy for eigenstates of the $X X X$ chain with finite density of particles, i.e., with finite $\rho \equiv M / L>0$ in the limit $L \gg 1$. To be specific here we present exact numerical data obtained from the Bethe ansatz solution of the model (see section 2) for a chain with $L=22$, focusing on $M=5$ and $M=7$. We restrict ourselves to 1 -strings and $\{M-2,1\}$-strings only 
(see section 2). Physically, the latter corresponds to eigenstates containing a single twoparticle bound state (2-string) and $M-2$ unbound particles. The construction of the Bethe ansatz wavefuction (6) for $\{M-2,1\}$-strings is outlined in Appendix C.

In sharp contrast with the situation with few particles (vanishing density), at finite $\rho$ the semiclassical upper bound $S_{M A X}(M)$ (cf. (5)) is no longer saturated. However, for highly-entangled eigenstates we provide numerical evidence that $S_{A}\left(L_{A}\right) \propto L_{c}$, with $L_{c} \equiv(L / \pi) \sin \left(\pi L_{A} / L\right)$ the chord length known from conformal field theory. Since $L_{c} \approx L_{A}$ for $L \gg L_{A}$ this also signals the volume law $S_{A}\left(L_{A}\right) \propto L_{A}$ (i.e., extensive entanglement). We also investigate how the entanglement entropy depends on the number of blocks of contiguous Bethe-Takahashi quantum numbers. We numerically observe that large number of blocks corresponds to high-entangled eigenstates (as in the low-density regime, see sections 3 and 4).

Finally, we consider eigenstates in the sector with $M=7$ containing a two-particle bound state. We find no significant change in the behavior of the von Neumann entropy, as compared to 1-string eigenstates.

\subsection{Extensive entanglement in 1-string eigenstates}

Figure 8 plots the von Neumann entropy $S_{A}\left(L_{A}\right)$ for eigenstates of the $X X X$ chain with $L=22$ in the sectors with $M=5$ and $M=7$ (panels (a)(b) and (c)(d), respectively). Here we restrict ourselves to 1-strings, i.e., real solutions of the Bethe equations (12). Data were obtained sampling over the Hilbert space of the $X X X$ chain using Monte Carlo (see Ref. [122] for the details of the Monte Carlo algorithm). Only $\sim 100$ eigenstates are shown in the figure. The dash-dotted line in panels (a)(c) is the semiclassical upper bound $S_{M A X}(M)$ (cf. (5)). Already in the sector with $M=5$, $S_{A}\left(L_{A}\right)$ shows deviations from $S_{M A X}(M)$, which become larger in the sector with $M=7$ (see Figure $8(\mathrm{c})$ ).

One should observe that at finite density $\rho$, in the large $L_{A} \ll L \operatorname{limit}, S_{M A X}(M)$ reduces to

$$
S_{M A X}(M)=\rho\left[1-\log \left(\frac{L_{A}}{L}\right)+\mathcal{O}\left(L_{A} / L\right)\right] L_{A}
$$

The term $\propto \log \left(L_{A} / L\right)$ in the prefactor in (32) diverges for $L_{A} / L \ll 1$, signaling that the semiclassical bound $S_{M A X}(M)$ exceeds the maximum allowed value $S_{A}\left(L_{A}\right)=L_{A} \log (2)$ for the von Neumann entropy.

Motivated by the result (3) for the ground state entanglement entropy of the $X X X$ chain [49], it is natural to assume that $S_{A}\left(L_{A}\right)$ is a function of the chord length $L_{c} \equiv(L / \pi) \sin \left(\pi L_{A} / L\right)$, i.e.,

$$
S_{A}\left(L_{A}\right)=f\left(L_{c}\right) \text {. }
$$

This is checked numerically in panels (b) and (d) for the eigenstates in the sector with $M=5$ and $M=7$, respectively. Clearly, $S_{A} \propto L_{c}$ for highly-entangled eigenstates. Since $L_{c} \approx L_{A}$ for $L_{A} \ll L$, the expected extensive behavior $S_{A}\left(L_{A}\right) \propto L_{A}$ is recovered. The full symbols (rhombi) in the figure show $S_{A}\left(L_{A}\right)$ for the eigenstates corresponding 

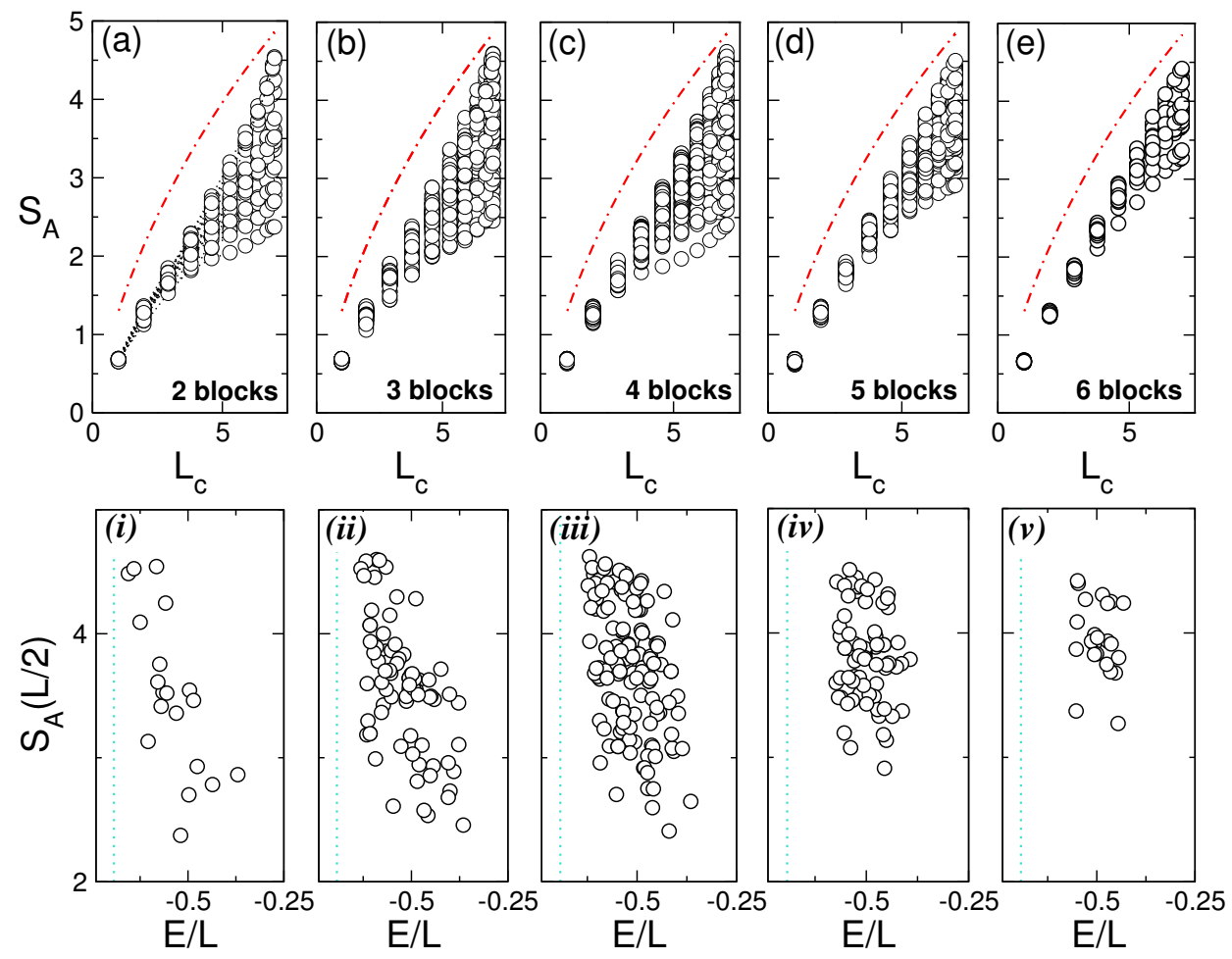

Figure 9. Entanglement entropy of the eigenstates of the $X X X$ chain in the sector with $M=7$. Entanglement entropy as a function of the number of blocks of contiguous occupied Bethe-Takahashi numbers (top panels) and the corresponding eigenstate energies $E$ (bottom panels). Data are exact numerics obtained from the Bethe ansatz solution of the model for a chain of size $L=22$. Here we restrict ourselves to 1-string eigenstates. (a)-(e) Entanglement entropy $S_{A}$ as a function of the chord length $L_{c} \equiv(L / \pi) \sin \left(\pi L_{A} / L\right)$, with $L_{A}$ the subsystem size. Different panels correspond to different numbers of Bethe number blocks: from two up to six blocks. In all panels the dashed line is the semiclassical upper bound $S_{M A X}(M=7)$. (i)(v) $S_{A}(L / 2)$ plotted versus the energy density $E / L$. The vertical dotted line denotes the ground state energy density $E_{g s} / L \approx-\log (2)$. Different panels are for different numbers of Bethe number blocks as in (a)-(e).

to Bethe-Takahashi numbers $\left[\circ \bigcirc^{4} \bullet \bigcirc^{2} \bullet \bigcirc^{3} \bigcirc^{2} \bullet\right]_{1}$ and $\left.\bullet^{2} \bigcirc^{4} \bigcirc \bullet \bullet \bigcirc^{2} \bullet^{2}\right]_{1}$ (see section 2 for the meaning of the notation). Clearly, a large number of blocks in the Bethe numbers is associated with linear behavior of the entanglement entropy.

The relation between entanglement and blocks of contiguous Bethe-Takahashi numbers is further investigated in Figure 9, considering the sector with $M=7$. The data are the same as in Figure 8. Panels (a)-(e) in the figure show the entanglement entropy as a function of the chord length $L_{c}$. Different panels correspond to the different numbers of blocks of contiguous Bethe quantum numbers: two blocks in panel (a), up to six in panel (e). The dash-dotted line in all panels is the semiclassical bound $S_{M A X}(M=7)$. Interestingly, already eigenstates with only two blocks give quite large values for the entanglement entropy. However, while $2 \lesssim S_{A}(L / 2) \lesssim 4.5$ for eigenstates in panel (a), one has $3 \lesssim S_{A}(L / 2) \lesssim 4.5$ in panel (e), meaning that eigenstates with many 

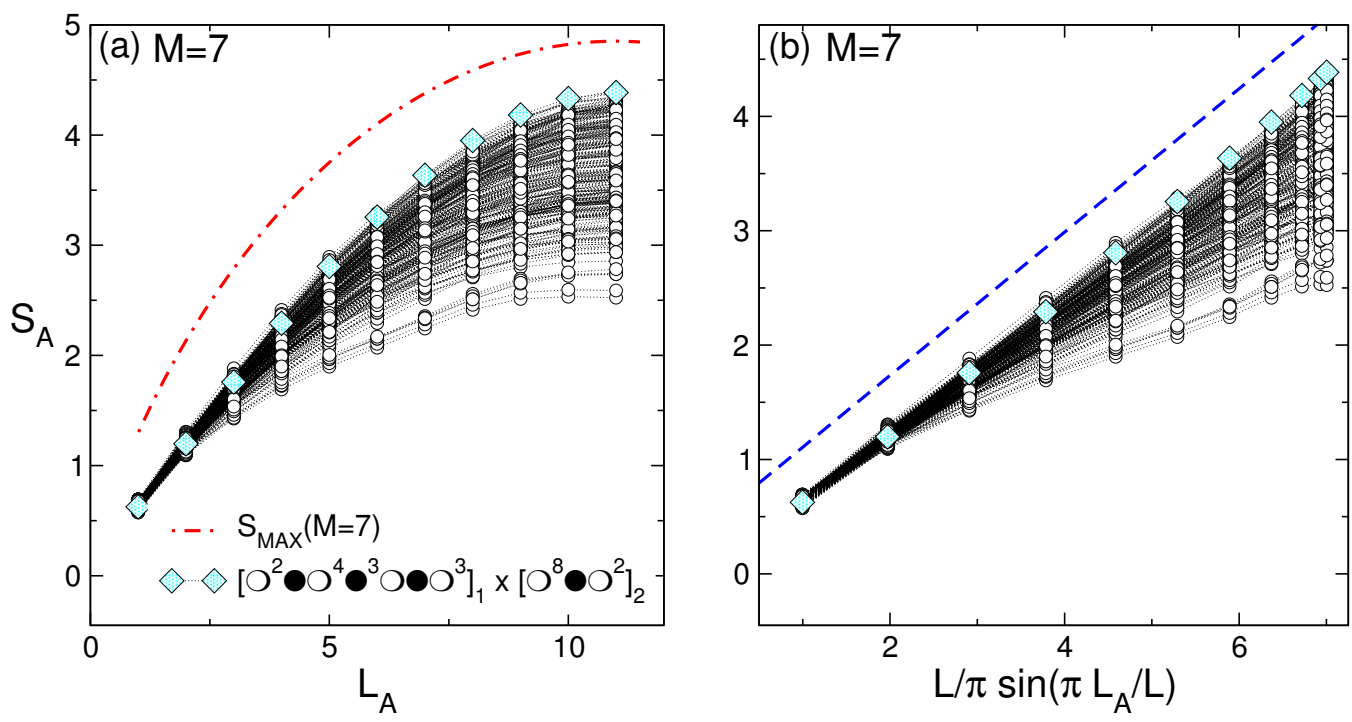

Figure 10. Crossover to the entropy volume law in the sector with $M=7$ particles of the $X X X$ chain. Here we focus on eigenstates containing only a single 2-string. Data are exact numerical results (Bethe ansatz) for a chain with $L=22$ sites ( $\sim 700$ eigenstates were considered). (a) Entanglement entropy $S_{A}\left(L_{A}\right)$ plotted versus the block length $L_{A}$. Full symbols (rhombi) denote $S_{A}$ for the eigenstate corresponding to $\left[\mathrm{O}^{2} \bigcirc^{4} \bullet^{3} \bigcirc \bigcirc^{3}\right]_{1} \times\left[\mathrm{O}^{8} \bullet \bigcirc^{2}\right]_{2}$, where $\bullet$ and $\bigcirc$ denote occupied and vacant Bethe quantum numbers, respectively. Here $[\cdots]_{\alpha}$ denote the BetheTakahashi quantum number configurations identifying the $\alpha$-string solutions of the Bethe-Takahashi equations. The dash-dotted line is the semiclassical bound $S_{M A X}(M)$ (same as in Figure 8). (b) Same data as in (a) plotted versus the chord length $L_{c} \equiv(L / \pi) \sin \left(\pi L_{A} / L\right)$. The dash-dotted line is a guide to the eye.

blocks typically correspond to highly-entangled states. Notice that one should expect a logarithmic entanglement scaling (similar to (3)) for eigenstates with few blocks, as shown in Ref. [70], while volume law should correspond to an extensive number of blocks. However, due to finite-size effects present for $L=22$, it is not possible to distinguish between the two behaviors in Figure 9.

Complementary information is shown in panels (i)-(v) where the half-chain entropy is plotted versus the eigenstate energy density $E / L \in[-\log (2), 0]$ [97] (same data as in (a)-(e), same scale is used on both axes in all panels). The vertical-dotted line denotes the ground-state energy density in the thermodynamic limit $E_{g s} / L=-\log (2)$. While eigenstates obtained from Bethe-Takahashi numbers with two blocks correspond to energies $-10 \lesssim E \lesssim 0$, many blocks correspond to a narrower energy window in the "bulk" of the spectrum. Also, notice that no clear band structure in the half-chain entropy is visible, in contrast with the low-density regime (cf. section 3 and 4). This is expected since the number of bands increases exponentially with $M$ (as $p(M)$ ), while the half-chain entropy is at most $S_{A} \propto M$. 


\subsection{Eigenstates containing only a single two-particle bound state}

The effects of bound states on the entanglement entropy of eigenstates of the $X X X$ chain with finite particle density $\rho$ are investigated in Figure 10. We focus on the $X X X$ chain with $L=22$ in the sector with $M=7$, restricting ourselves to eigenstates with a single two-particle bound state.

In panel (a) the von Neumann entropy $S_{A}\left(L_{A}\right)$ is plotted versus the block length $L_{A}$. The dash-dotted line is the semiclassical bound $S_{M A X}(M)$ (as in Figure 8). Interestingly, the behavior of the entropy is not affected significantly by the two-particle bound state, and $2.5 \lesssim S_{A}(L / 2) \lesssim 4.5$, as for 1-string eigenstates (compare with Figure 8). The full rhombi show the von Neumann entropy for the (highly-entangled) eigenstate obtained from Bethe-Takahashi quantum numbers $\left[\bigcirc^{2} \bigcirc^{4} \bigcirc^{3} \bigcirc \bigcirc^{3}\right]_{1} \times\left[\bigcirc^{8} \bigcirc^{2}\right]_{2}$, with $[\cdots]_{1}$ and $[\cdots]_{2}$ denoting the quantum numbers for the 1-strings and the 2-string, respectively (see section 2). The same data are shown in panel (b) versus the chord length $L_{c} \equiv(L / \pi) \sin \left(\pi L_{A} / L\right)$. Highly-entangled eigenstates exhibit extensive entanglement entropy, as for the 1-string eigenstates.

\section{Conclusions}

In this paper, by exploiting the Bethe ansatz solution of the model [96,97], we investigated the entanglement entropy and entanglement spectra (ES) of the eigenstates of the spin- $\frac{1}{2}$ isotropic Heisenberg chain ( $X X X$ chain). In contrast with Ref. [70], which focused on eigenstates corresponding to real solutions (rapidities) of the Bethe equations, here we considered both real (1-strings) and complex rapidities ( $n$-strings, with $n \in \mathbb{N}$, $n>1$ ). While the former are states of interacting magnons, $n$-strings signal the presence of many-particle bound states. We addressed both the situations with low and finite particle density $\rho \equiv M / L, M$ and $L$ being the particle number and the chain length, respectively.

We first considered the situation with $M=2$ and $M=3$ (i.e., few particles in the chain). For $M=2$, based on the Bethe ansatz results, we derived an upper bound $S_{M A X}$ for the entanglement entropy. Using semiclassical arguments this was generalized for arbitrary $M$ as

$$
S_{M A X}(M)=-M(\omega \log (\omega)+(1-\omega) \log (1-\omega)),
$$

with $\omega$ the ratio $\omega \equiv L_{A} / L$, and $L_{A}$ the length of subsystem $A$. For $M=1$, (34) is the entanglement entropy of a free particle. The linear dependence $S_{A}(M) \propto M$ reflects that in the semiclassical approximation the interactions between particles can be neglected. For $L_{A} / L \ll 1, S_{M A X} \approx-\left(M L_{A} / L\right) \log \left(L_{A} / L\right)$, which is an "intermediate" behavior between logarithmic and extensive. Remarkably, for highly-entangled 1-string eigenstates with $M=2$ (and in general in the vanishing density regime, i.e., at fixed $M$ and $L \rightarrow \infty) S_{M A X}(M)$ is saturated, i.e., $S_{A} \approx S_{M A X}$, apart from $\mathcal{O}(1 / L)$ terms.

Focusing on the half-chain entanglement entropy, we investigated the relation between entanglement and eigenstate energy. In the vanishing density regime, the half-chain 
entropy exhibits a "band" structure, when plotted against energy. This is understood in terms of the Bethe-Takahashi quantum numbers, which identify the eigenstates of the $X X X$ chain. Specifically, higher entanglement bands correspond to states with a larger number of blocks of contiguous Bethe-Takahashi numbers. The number of entanglement bands, which is obtained by counting the possible ways of grouping the quantum numbers, is given by the integer partitions $p(M)$ of $M$. For eigenstates with maximum number of Bethe number blocks it is $S_{A} \approx S_{M A X}$. Interestingly, the corresponding entanglement spectrum (ES) levels are simple functions of the ratio $\omega \equiv L_{A} / L$ that are understood in the semiclassical picture.

For eigenstates that contain bound states, the entanglement entropy is dramatically reduced, as compared to the situation with unbound particles only. Since bound states can be treated effectively as single particles, this reflects a reduction in the number of degrees of freedom. The finite-size behavior of the entanglement entropy is quantitatively characterized in terms of the bound-state extension $\ell_{b}$, which is an increasing function of the bound-state energy, and it is known analytically from the Bethe ansatz solution. Strongly-bound (equivalently, low-energy) and weakly-bound (i.e., high-energy) particles correspond to $\ell_{b} / L \ll 1$ and $\ell_{b} / L \gg 1$, respectively. Clearly, the entanglement entropy increases upon increasing $\ell_{b} \lesssim L$, i.e., as particles become weakly bound. The presence of bound states has striking effects at the level of the half-chain entanglement spectrum (ES). Specifically, we demonstrated that the ES exhibits edge-related levels. The corresponding entanglement eigenfunctions (i.e., the eigenvectors of the reduced density matrix) are exponentially localized at the boundary between the two subsystems.

This semiclassical scenario outlined above breaks down at finite particle density. A striking change is that the semiclassical upper bound $S_{M A X}$ is not saturated. Interestingly, for highly-entangled eigenstates it is now $S_{A}\left(L_{A}\right) \propto L_{c}$, with $L_{c}$ the chord length $L_{c} \equiv(L / \pi) \sin \left(\pi L_{A} / L\right)$. Since $L_{c} \approx L_{A}$ for small subsystems, this signals the extensive entanglement. We also investigated the relation between entanglement and the number of blocks of contiguous Bethe-Takahashi numbers. As for vanishing density, eigenstates corresponding to a large number of blocks are highly-entangled. Finally, we studied the effects of bound states, focusing on eigenstates with $\rho \approx 1 / 3$ and only one two-particle bound state. We provided numerical evidence that the presence of the bound state does not affect significantly the entanglement entropy.

\section{Appendix A. Entanglement properties of two unbound particles}

In this section we focus on the eigenstates of the $X X X$ chain in the sector with two particles, i.e., with $M=2$. We derive analytically the entanglement spectrum (ES) and the entanglement entropy for a generic wavefunction $\left|\Psi_{2}\right\rangle$ of the form (6).

As a function of the two quasi-momenta $k_{1}$ and $k_{2},\left|\Psi_{2}\right\rangle$ reads

$$
\left|\Psi_{2}\right\rangle=\frac{1}{\mathcal{N}} \sum_{x_{1}<x_{2}}\left[e^{i k_{1} x_{1}+i k_{2} x_{2}+i \theta_{1,2}}+e^{i k_{1} x_{2}+i k_{2} x_{1}+i \theta_{2,1}}\right]\left|x_{1}, x_{2}\right\rangle,
$$


where $\left|x_{1}, x_{2}\right\rangle$ denotes the configuration with the two particles at positions $x_{1}, x_{2}$ in the chain, and the normalization $\mathcal{N}$ is given as

$$
\mathcal{N}=\left[L(L-1)+\frac{-L \cos (k-\theta)+\cos [L k-\theta]+(L-1) \cos (\theta)}{\cos (k)-1}\right]^{\frac{1}{2}} .
$$

Here $k \equiv k_{1}-k_{2}$ is the relative quasi-momentum between the two particles, and $\theta \equiv \theta_{2,1}-\theta_{1,2}$ the scattering phase (cf. (8)).

In order to derive entanglement properties it is useful to introduce the Schmidt matrix $\mathbb{M}(A \mid B)$. Given a generic wavefunction $|\Psi\rangle$ and a bipartition of a system into two parts $A$ and $B, \mathbb{M}(A \mid B)$ is defined such that

$$
|\Psi\rangle=\sum_{i, j} \mathbb{M}_{i, j}(A \mid B)\left|\varphi_{i}^{(A)}\right\rangle \otimes\left|\varphi_{j}^{(B)}\right\rangle,
$$

where $\left|\varphi_{i}^{(A)}\right\rangle$ and $\left|\varphi_{i}^{(A)}\right\rangle$ form two orthonormal bases for part $A$ and $B$, respectively. Notice that the Schmidt decomposition (1) corresponds to the singular value decomposition (SVD) of $\mathbb{M}_{i, j}(A \mid B)$. In particular, the non-zero singular values $\zeta_{i}$ of $\mathbb{M}(A \mid B)$ are related to the reduced-density-matrix (for part $A$ ) eigenvalues $\lambda_{i}$ as $\lambda_{i}=\zeta_{i}^{2}$. Here for the case with $|\Psi\rangle=\left|\Psi_{2}\right\rangle$ (cf. (A.1)), the orthonormal basis $\left\{\left|\varphi_{i}^{(A)}\right\rangle\right\}$ for $A$ can be chosen as

$$
\begin{aligned}
& \left|\Omega_{A}\right\rangle \equiv|\uparrow \uparrow \cdots \uparrow\rangle_{A} \\
& \left|\varphi_{A}\right\rangle=\frac{1}{\mathcal{N}_{A}} \sum_{x_{1}<x_{2} \in A}\left[e^{i k_{1} x_{1}+i k_{2} x_{2}+i \theta_{1,2}}+e^{i k_{1} x_{2}+i k_{2} x_{1}+i \theta_{2,1}}\right]\left|x_{1}, x_{2}\right\rangle_{A} \\
& \left|\varphi_{A}^{\prime}\left(k_{1}\right)\right\rangle=\frac{1}{\sqrt{L_{A}}} \sum_{x \in A} e^{i k_{1} x}|x\rangle_{A} \\
& \left|\varphi_{A}^{\prime \prime}\left(k_{1}, k_{2}\right)\right\rangle=\frac{1}{\sqrt{1-\left|\Gamma_{A}\right|^{2}}}\left[\left|\varphi_{A}^{\prime}\left(k_{2}\right)\right\rangle-\Gamma_{A}\left|\varphi_{A}^{\prime}\left(k_{1}\right)\right\rangle\right]
\end{aligned}
$$

where $L_{A}$ is the length of subsystem $A$, and $|x\rangle_{A} \equiv S_{x}^{-}\left|\Omega_{A}\right\rangle,\left|x_{1}, x_{2}\right\rangle_{A} \equiv S_{x_{1}}^{-} S_{x_{2}}^{-}\left|\Omega_{A}\right\rangle$. In (A.4) $\mathcal{N}_{A}$ is obtained from (A.2) after replacing $L \rightarrow L_{A}$, and the overlap $\Gamma_{A}$ reads

$$
\Gamma_{A} \equiv\left\langle\varphi_{A}^{\prime}\left(k_{1}\right) \mid \varphi_{A}^{\prime}\left(k_{2}\right)\right\rangle=\frac{1}{L_{A}} \frac{\sin \frac{k}{2} L_{A}}{\sin \frac{k}{2}} \exp \left[-i \frac{k}{2}\left(1+L_{A}\right)\right] .
$$

The basis $\left\{\left|\varphi_{i}^{(B)}\right\rangle\right\}$ for subsystem $B$ is obtained from (A.4) by replacing $A \rightarrow B$, and using that

$$
\Gamma_{B}=\frac{1}{L_{B}} \frac{\sin \frac{k}{2} L_{B}}{\sin \frac{k}{2}} \exp \left[-i \frac{k}{2}\left(1+L_{B}\right)-i k L_{A}\right] .
$$

The Bethe wavefunction $\left|\Psi_{2}\right\rangle$ is then rewritten as

$$
\begin{aligned}
& \left|\Psi_{2}\right\rangle=\frac{1}{\mathcal{N}}\left[\mathcal{N}_{A}\left|\varphi_{A}\right\rangle \otimes\left|\Omega_{B}\right\rangle+\mathcal{N}_{B}\left|\Omega_{A}\right\rangle \otimes\left|\varphi_{B}\right\rangle+\sqrt{L_{A} L_{B}\left(1-\left|\Gamma_{B}\right|^{2}\right)}\left|\varphi_{A}^{\prime}\right\rangle \otimes\left|\varphi_{B}^{\prime \prime}\right\rangle\right. \\
& \left.+e^{i \theta} \sqrt{L_{A} L_{B}\left(1-\left|\Gamma_{A}\right|^{2}\right)}\left|\varphi_{A}^{\prime \prime}\right\rangle \otimes\left|\varphi_{B}^{\prime}\right\rangle+\sqrt{L_{A} L_{B}}\left(\Gamma_{B}+\Gamma_{A} e^{i \theta}\right)\left|\varphi_{A}^{\prime}\right\rangle \otimes\left|\varphi_{B}^{\prime}\right\rangle\right] .
\end{aligned}
$$


The resulting Schmidt matrix (A.3) reads

$\mathbb{M}(A \mid B)=\frac{1}{\mathcal{N}}\left[\begin{array}{cccc}0 & \mathcal{N}_{B} & 0 & 0 \\ \mathcal{N}_{A} & 0 & 0 & 0 \\ 0 & 0 & \left(L_{A} L_{B}\right)^{1 / 2}\left(\Gamma_{B}+e^{i \theta} \Gamma_{A}\right) & \left(L_{A} L_{B}\left(1-\left|\Gamma_{B}\right|^{2}\right)\right)^{1 / 2} \\ 0 & 0 & e^{i \theta}\left(L_{A} L_{B}\left(1-\left|\Gamma_{A}\right|^{2}\right)\right)^{1 / 2} & 0\end{array}\right]$

The two entries in the upper-left $2 \times 2$ block in (A.7) correspond to both particles being in subsystem $A$ and subsystem $B$, respectively. The bottom-right block corresponds to having one particle in subsystem $A$ and one in $B$. The singular values decompositions of (A.7) is straightforward. $\mathbb{M}(A \mid B)$ has four non-zero singular values. Two singular values are $\zeta_{0}=\mathcal{N}_{A} / \mathcal{N}$ and $\zeta_{0}^{\prime}=\mathcal{N}_{B} / \mathcal{N}$. The remaining ones $\zeta_{ \pm}$are given as

$\zeta_{ \pm}=\frac{1}{\mathcal{N}}\left[L_{A} L_{B}\left(1+\operatorname{Re}\left(\Gamma_{B}^{*} \Gamma_{A} e^{i \theta}\right) \pm \sqrt{\left(1+\operatorname{Re}\left(\Gamma_{A}^{*} \Gamma_{B} e^{-i \theta}\right)\right)^{2}-\left(\left|\Gamma_{A}\right|^{2}-1\right)\left(\left|\Gamma_{B}\right|^{2}-1\right)}\right)\right]^{\frac{1}{2}}$

In the limit $L_{A}, L_{B} \gg 1$ from (A.5) and (A.6) one has that $\Gamma_{A} \propto 1 / L_{A}$ and $\Gamma_{B} \propto 1 / L_{B}$. The singular values of $\mathbb{M}(A \mid B)$ become $\zeta_{0} \approx \omega, \zeta_{0}^{\prime} \approx 1-\omega$, and $\zeta_{+} \approx \zeta_{-} \approx \omega^{\frac{1}{2}}(1-\omega)^{\frac{1}{2}}$, with $\omega$ being the ratio $\omega \equiv L_{A} / L$. These are related to the "semiclassical" probabilities of distributing the particles in subsystem $A$ and $B$. We mention that similar results are obtained for eigenstates with $M>2$ (see Appendix D). Notice that in the semiclassical approximation the scattering phase $\theta=\theta_{2,1}-\theta_{1,2}$ is irrelevant, as expected. However, this semiclassical approximation holds at finite (i.e., non-vanishing) relative quasimomentum $k \equiv k_{2}-k_{1}$. For $k \rightarrow 0, \theta$ cannot be neglected, and the outlined picture breaks down. The limit $k \rightarrow 0$ is more carefully discussed in the next subsection.

\section{Appendix A.1. Low relative quasi-momentum limit}

In this section we derive the entanglement entropy and entanglement spectrum for the two-particle wavefunction (A.1) assuming $k \rightarrow 0$, i.e., small relative quasi-momentum between the two particles. Specifically, we assume $k_{2}-k_{1}=2 \pi \frac{\alpha}{L}$ and $\alpha=\mathcal{O}(1)$. Using (11) and the logarithmic Bethe equations (12) one obtains

$$
k_{2}-k_{1}=2 \pi \frac{\delta J}{L}+\frac{4}{L} \arctan \left(\lambda_{1}-\lambda_{2}\right), \quad \text { with } \quad \delta J \equiv J_{2}-J_{1}
$$

Since $\lambda_{1} \rightarrow \lambda_{2}$ in the limit $L \rightarrow \infty, \alpha \approx \delta J$, which justifies the assumption $\alpha=\mathcal{O}(1)$. Substituting in (A.5)(A.6), one obtains

$$
\Gamma_{A} \approx \frac{\sin \pi \alpha \omega}{\pi \alpha \omega} e^{-i \pi \alpha \omega}, \quad \Gamma_{B} \approx \frac{\sin \pi \alpha(1-\omega)}{\pi \alpha(1-\omega)} e^{-i \pi \alpha(1+\omega)}
$$

where $\omega \equiv L_{A} / L$, and the limit $L \gg 1$ was taken. The two singular values $\zeta_{0}, \zeta_{0}^{\prime}$ of the Schmidt matrix $\mathbb{M}(A \mid B)$ (cf. (A.7)) become

$$
\begin{aligned}
& \zeta_{0}=\left[\frac{2(\pi \alpha \omega)^{2}-\cos (2 \pi \alpha \omega+\theta)-\cos (\theta)}{2(\pi \alpha)^{2}-\cos (2 \pi \alpha+\theta)-\cos (\theta)}\right]^{\frac{1}{2}} \\
& \zeta_{0}^{\prime}=\left[\frac{2(\pi \alpha(1-\omega))^{2}-\cos (2 \pi \alpha(1-\omega)+\theta)-\cos (\theta)}{2(\pi \alpha)^{2}-\cos (2 \pi \alpha+\theta)-\cos (\theta)}\right]^{\frac{1}{2}}
\end{aligned}
$$


Notice that $\theta$ is not negligible unless the limit $\alpha \rightarrow \infty$ is taken. Similar results can be obtained for $\zeta_{ \pm}$using (A.9) (A.5)(A.6) and (cf. (A.2))

$$
\mathcal{N}=L\left[1-\frac{\cos (2 \pi \alpha+\theta)-\cos (\theta)}{(2 \pi \alpha)^{2}}\right]^{\frac{1}{2}} .
$$

The entanglement entropy obtained in the low relative quasi-momentum limit is reported as dotted line in Figure 2.

\section{Appendix B. Bethe wavefunctions for many-particle bound states}

In this section we focus on eigenstates of the $X X X$ chain in the sectors with $M=2,3$, focusing on 2-strings and 3-strings solutions of the Bethe-Takahashi equations (20), respectively. Physically, this corresponds to the situation in which all the particles form a bound state. Here we first discuss how to treat the singularities appearing in the wavefunction amplitudes (6), due to the presence of strings. We then derive exact results for the entanglement entropy and the entanglement spectrum.

\section{Appendix B.1. Two-particle bound states}

Here we restrict ourselves to two-particle bound states, i.e., $M=2$. Using the string hypothesis (28), the two rapidities $\lambda_{1}, \lambda_{2}$ identifying the eigenstates of the $X X X$ chain read

$$
\lambda_{1}=\lambda-i-i \delta, \quad \lambda_{2}=\lambda+i+i \delta .
$$

Notice that in (B.1) we take into account the finite-size deviations $\delta=\mathcal{O}\left(e^{-L}\right)$ from the string hypothesis (see section 2). In the limit $L \rightarrow \infty$ the string center $\lambda$ is determined by solving the Bethe-Takahashi equations (20). The Bethe ansatz wavefunction for two particles reads (cf. (6))

$$
\left|\Psi_{2}\right\rangle=\frac{1}{\mathcal{N}} \sum_{x_{1}<x_{2}}\left[e^{i k_{1} x_{1}+i k_{2} x_{2}+i \theta_{1,2}}+e^{i k_{1} x_{2}+i k_{2} x_{1}+i \theta_{2,1}}\right]\left|x_{1}, x_{2}\right\rangle,
$$

with $k_{1}, k_{2}$ the two quasi-momenta obtained from (B.2) using (24). From (29) the scattering phases $\theta_{1,2}$ and $\theta_{2,1}$ are given as

$$
\begin{aligned}
& \theta_{1,2}=\frac{1}{2 i} \log \left[1+\frac{4}{\hat{\delta}}\right] \rightarrow-\frac{1}{2 i} \log (\hat{\delta}) \\
& \theta_{2,1}=\frac{1}{2 i} \log \left[1+\frac{4}{4-\hat{\delta}}\right] \rightarrow \frac{1}{2 i} \log (\hat{\delta}),
\end{aligned}
$$

with $\hat{\delta}=2 \delta$. The limit $\delta \rightarrow 0$ (equivalently $L \rightarrow \infty$ ) was taken in the last step in (B.3). Using (B.1) and (B.3) the wavefunction B.2 becomes

$$
\left|\Psi_{2}\right\rangle=\frac{1}{\mathcal{N}} \sum_{x_{1}<x_{2}}\left[e^{i K_{b}\left(x_{1}+x_{2}\right)}\left(\frac{1}{\sqrt{\delta}} e^{-\left(x_{2}-x_{1}\right) / \ell_{b}}+\sqrt{\delta} e^{\left(x_{2}-x_{1}\right) / \ell_{b}}\right)\right]\left|x_{1}, x_{2}\right\rangle,
$$

with $K_{b} \equiv k_{1}+k_{2} \in \mathbb{R}$ the total momentum of the bound state, and $\ell_{b}$ the bound-state length ( (27) with $n=2$ ). Notice that, although the second term in (B.4) vanishes 
exponentially for $L \rightarrow \infty$, it cannot be neglected when $x_{2}-x_{1} \rightarrow \infty$. Also, neglecting the second term would lead to the inconsistency $\Psi_{2}\left(x_{1}=1, x_{2}=2\right) \neq \Psi_{2}\left(x_{1}=1, x_{2}=\right.$ $L-1)$. However, the Bethe equations (10) imply that

$$
\theta_{2,1}=\theta_{1,2}-\left(k_{1}-k_{2}\right) \frac{L}{2} \quad \bmod \pi
$$

Substituting (B.5) in (B.4), one obtains

$$
\left|\Psi_{2}\right\rangle \propto e^{i \theta_{1,2}} \sum_{x_{1}<x_{2}} e^{i K_{b}\left(x_{1}+x_{2}\right)}\left[e^{-\left(x_{2}-x_{1}\right) / \ell_{b}}+e^{\left(x_{2}-x_{1}-L\right) / \ell_{b}}\right]\left|x_{1}, x_{2}\right\rangle .
$$

Notice that all the terms inside the brackets in (B.6) are now regular. The overall divergent factor $e^{i \theta_{1,2}}$ cancels when normalizing the wavefunction.

Entanglement properties. In order to derive the entanglement entropy for the twoparticle bound state, here we consider the simplified situation in which we neglect the second term in (B.6). The consequences of this approximation are discussed at the end of the section. The two-particle bound state wavefunction (B.6) reads

$$
\left|\Phi_{2}\right\rangle=\frac{1}{\mathcal{N}} \sum_{x_{1}<x_{2}} e^{i K_{b}\left(x_{1}+x_{2}\right)} e^{-\left(x_{2}-x_{1}\right) / \ell_{b}}\left|x_{1}, x_{2}\right\rangle
$$

The normalization $\mathcal{N}$ does not depend on the total momentum $K_{b}$ and it is given as

$$
\mathcal{N} \approx \frac{\left(-L+e^{2 / \ell_{b}}(L-1)\right)^{\frac{1}{2}}}{e^{2 / \ell_{b}}-1},
$$

where we neglected $\mathcal{O}\left(e^{-L}\right)$ terms. To construct the Schmidt matrix $\mathbb{M}(A \mid B)$ (cf. (A.3)) we choose the orthonormal basis for $A$ as

$$
\begin{aligned}
\left|\Omega_{A}\right\rangle & \equiv|\uparrow \uparrow \cdots \uparrow\rangle_{A} \\
\left|\varphi_{A}\right\rangle & \equiv \frac{1}{\mathcal{N}_{A}} \sum_{x_{1}<x_{2} \in A} e^{i K_{b}\left(x_{1}+x_{2}\right)} e^{-\left(x_{2}-x_{1}\right) / \ell_{b}}\left|x_{1}, x_{2}\right\rangle_{A} \\
\left|\varphi_{A}^{\prime}\right\rangle & \equiv \frac{1}{\mathcal{N}_{A}^{\prime}} \sum_{x \in A} e^{i K_{b} x+x / \ell_{b}}|x\rangle_{A} .
\end{aligned}
$$

The basis for the $B$ part can be chosen analogously. The Schmidt matrix then reads

$$
\mathbb{M}(A \mid B)=\frac{1}{\mathcal{N}}\left[\begin{array}{ccc}
0 & \mathcal{N}_{B} & 0 \\
\mathcal{N}_{A} & 0 & 0 \\
0 & 0 & \mathcal{N}_{A}^{\prime} \mathcal{N}_{B}^{\prime}
\end{array}\right]
$$

where

$$
\begin{array}{ll}
\mathcal{N}_{A} \approx \frac{\left(-L_{A}+e^{2 / \ell_{b}}\left(L_{A}-1\right)\right)^{\frac{1}{2}}}{e^{2 / \ell_{b}}-1}, & \mathcal{N}_{B} \approx \frac{\left(-L_{B}+e^{2 / \ell_{b}}\left(L_{B}-1\right)\right)^{\frac{1}{2}}}{e^{2 / \ell_{b}}-1}, \\
\mathcal{N}_{A}^{\prime} \approx \frac{e^{L_{A} / \ell_{b}}}{\left(1-e^{-2 / \ell_{b}}\right)^{\frac{1}{2}}}, & \mathcal{N}_{B}^{\prime} \approx \frac{e^{-L_{A} / \ell_{b}}}{\left(e^{2 / \ell_{b}}-1\right)^{\frac{1}{2}}}
\end{array}
$$


In (B.11) $\mathcal{O}\left(e^{-L / \ell_{b}}\right)$ terms have been neglected. $\mathbb{M}(A \mid B)$ has three non-zero singular values $\zeta_{0}=\mathcal{N}_{A} / \mathcal{N}, \zeta_{0}^{\prime}=\mathcal{N}_{B} / \mathcal{N}$, and $\zeta \equiv \mathcal{N}_{A}^{\prime} \mathcal{N}_{B}^{\prime} / \mathcal{N}$, which read

$$
\begin{aligned}
& \zeta_{0} \approx\left[\frac{-L_{A}+e^{2 / \ell_{b}}\left(L_{A}-1\right)}{-L+e^{2 / \ell_{b}}(L-1)}\right]^{\frac{1}{2}}, \quad \zeta_{0}^{\prime} \approx\left[\frac{-L_{B}+e^{2 / \ell_{b}}\left(L_{B}-1\right)}{-L+e^{2 / \ell_{b}}(L-1)}\right]^{\frac{1}{2}}, \\
& \zeta \approx\left[\frac{e^{2 / \ell_{b}}}{-L+e^{2 / \ell_{b}}(L-1)}\right]^{\frac{1}{2}} .
\end{aligned}
$$

Clearly, $\zeta_{0} \rightarrow \omega^{\frac{1}{2}}$ and $\zeta^{\prime} \rightarrow(1-\omega)^{\frac{1}{2}}$ for $L \rightarrow \infty$, whereas $\zeta$ is vanishing as $\propto 1 / L^{\frac{1}{2}}$. Notice that $\zeta$ corresponds to a diverging entanglement spectrum level $\xi \equiv-2 \log (\zeta)$. Interestingly, while $\zeta_{0}, \zeta_{0}^{\prime}$ are usual "bulk" singular values, similar to the case with two unbound particles (see Appendix A), $\zeta$ corresponds to an edge-related one. This is clear from the associated singular vector $\left|\varphi_{A}^{\prime}\right\rangle$ (cf. (B.9)), which is exponentially localized at the boundary of part $A$.

It is interesting to rewrite (B.12) in terms of the energy $E$ (cf. (25)). Here we restrict to the case with part $A$ being half of the chain. Using (27) one obtains

$$
\zeta_{0}=\zeta_{0}^{\prime}=\left[\frac{2+L E-2(1+E)^{L / 2}}{2+L E-2(1+E)^{L}}\right]^{\frac{1}{2}}, \quad \zeta=\frac{-1+(1+E)^{L / 2}}{\left(-1-L E+(1+E)^{L}\right)^{\frac{1}{2}}} .
$$

From (B.12) the half-chain entropy reads

$$
S_{A}(L / 2)=\log (2)-\frac{-1+\log (2)-\log \left(1-e^{-2 / \ell_{b}}\right)-\log (L)}{1-e^{-2 / \ell_{b}}} \frac{1}{L}+\mathcal{O}\left(1 / L^{2}\right),
$$

and, in terms of the energy $E$, one has

$$
S_{A}(L / 2)=\log (2)-\frac{1}{L E}[1-\log (2)+\log (L)+\log (-E)]+\mathcal{O}\left(1 / L^{2}\right) .
$$

Clearly, in the limit $L \rightarrow \infty, S_{A}(L / 2) \approx \log (2)$. For fixed $L$, high-energy bound states exhibit deviations from $S_{A}(L / 2)=\log (2)$. This correspond to eigenstates containing two weakly-bound particles (cf. (27)). In the limit $E \rightarrow 0$ (equivalently $\ell_{b} \rightarrow \infty$ ), the second term in (B.15) diverges. Notice that this is a spurious divergence due to the fact that we neglected $\mathcal{O}\left(e^{-L / \ell_{b}}\right)$ terms in (B.12).

We should stress that the results outlined above are not strongly affected by the second term in (B.6). The only significant change is that, while the Schmidt matrix obtained from (B.7) has only one edge-related singular value ( $\zeta$ in (B.13)), an extra one $\zeta^{\prime} \approx \zeta$ appears if one considers (B.6). This is due to the fact that subsystem $A$ has two boundaries. Clearly, $\zeta$ and $\zeta^{\prime}$ correspond to a particle localized at the right and left edge of $A$, respectively. Similarly, taking into account the second term in (B.6) the expression for the half-chain entropy (B.15) is modified as

$$
S_{A}(L / 2)=\log (2)-\frac{2}{L E}[1-\log (2)+\log (L)+\log (-E)]+\mathcal{O}\left(1 / L^{2}\right) .
$$

Notice the factor two (reflecting the number of boundaries of block $A$ ) in the $\mathcal{O}(1 / L)$ term. 
Appendix B.2. Three-particle bound states

Here we discuss the structure of the Bethe wavefunction (6) for the 3-particle bound states. These correspond to three solutions $\Lambda=\left\{\lambda_{1}, \lambda_{2}, \lambda_{3}\right\}$ of the Bethe equations (10) forming a (deviated) 3-string in the complex plane as

$$
\Lambda=\left\{\begin{array}{l}
\lambda_{1}=\lambda+\epsilon+2 i+i \delta \\
\lambda_{2}=\lambda+\epsilon-2 i-i \delta \\
\lambda_{3}=\lambda
\end{array}\right.
$$

where $\delta, \epsilon$ (string deviations) are $\mathcal{O}\left(e^{-L}\right)$. The string center $\lambda \in \mathbb{R}$ is obtained by solving the Bethe-Takahashi equations (20). Using (29) one obtains $\theta_{1,3} \rightarrow-i \infty$ and $\theta_{2,3} \rightarrow$ $+i \infty$ in the limit $\delta \rightarrow 0$ (equivalently $L \rightarrow \infty$ ). The only permutations of $\left\{\lambda_{1}, \lambda_{2}, \lambda_{3}\right\}$ leading to singularities in the wavefunction amplitude $(7)$ are $\{1,3,2\},\{2,3,1\},\{2,1,3\}$, and $\{2,3,1\}$. Clearly, the "most divergent" amplitude in $(6)$ is $\propto \exp \left(i \theta_{1,3}+i \theta_{3,2}\right)$, which corresponds to $\{1,3,2\}$. The remaining two permutations $\{1,2,3\}$ and $\{3,2,1\}$ contribute with finite terms that can be neglected. Using the Bethe equations (10) one obtains a relation between $\theta_{1,3}, \theta_{3,2}$ and $\theta_{3,1}, \theta_{2,3}$ (similar to (B.5)) as

$$
i\left(k_{1}-3 k_{2}-k_{3}\right) \frac{L}{2}+i \theta_{3,1}+i \theta_{2,3}-4 i \theta_{1,2}-i \theta_{1,3}-i \theta_{3,2}=0 \quad \bmod \pi .
$$

Using (B.17) the Bethe wavefunction (6) reads

$$
\begin{aligned}
\left|\Psi_{3}\right\rangle \propto \exp \left(2 i \theta_{1,3}\right) & \sum_{x_{1}<x_{2}<x_{3}}\{ \\
& \exp \left[i K_{b}\left(x_{1}+x_{3}\right)+i k_{3} x_{2}-\left(x_{3}-x_{1}\right) / \ell_{b}\right]+ \\
& \exp \left[i K_{b}\left(x_{1}+x_{2}\right)+i k_{3} x_{3}+\left(x_{2}-x_{1}-L\right) / \ell_{b}+i K_{T} L / 4\right]+ \\
& \left.\exp \left[i K_{b}\left(x_{2}+x_{3}\right)+i k_{3} x_{1}+\left(x_{3}-x_{2}-L\right) / \ell_{b}+i K_{T} L / 4\right]\right\}\left|x_{1}, x_{2}, x_{3}\right\rangle
\end{aligned}
$$

with $\ell_{b}$ the bound-state length obtained from (27), $K_{b} \equiv k_{1}+k_{2} \in \mathbb{R}$, and $K_{T} \equiv$ $k_{1}+k_{2}+k_{3} \in \mathbb{R}$ the total momentum of the eigenstate. Since $x_{1}<x_{2}<x_{3}$, the first term in (B.18) corresponds to a three-particle bound state, whereas the other two are superpositions of a two-particle bound state and a free magnon. Notice that the overall divergent term $\exp \left(2 i \theta_{1,3}\right)$ in (B.18) cancel in the normalized wavefunction.

Entanglement properties. To discuss the entanglement spectrum and entanglement entropy of the three-particle bound state, we consider the simplified situation in which we keep only the first amplitude in (B.18), i.e.,

$$
\left|\Psi_{3}\right\rangle=\frac{1}{\mathcal{N}} \sum_{x_{1}<x_{2}<x_{3}} e^{-\left(x_{3}-x_{1}\right) / \ell_{b}} e^{i K_{b}\left(x_{1}+x_{3}\right)+i k_{3} x_{2}}\left|x_{1}, x_{2}, x_{3}\right\rangle .
$$

The normalization of (B.19) in the large $L$ limit is

$$
\mathcal{N} \approx \frac{\left(e^{2 / \ell_{b}}(L-2)-L\right)^{\frac{1}{2}}}{\left(e^{2 / \ell_{b}}-1\right)^{\frac{3}{2}}}
$$


The Schmidt matrix $\mathbb{M}(A \mid B)$ from (B.19) can be given as

$$
\mathbb{M}(A \mid B)=\frac{1}{\mathcal{N}}\left[\begin{array}{cccc}
0 & \mathcal{N}_{B} & 0 & 0 \\
\mathcal{N}_{A} & 0 & 0 & 0 \\
0 & 0 & 0 & \mathcal{N}_{A}^{\prime \prime} \mathcal{N}_{B}^{\prime} \\
0 & 0 & \mathcal{N}_{A}^{\prime} \mathcal{N}_{B}^{\prime \prime} & 0
\end{array}\right]
$$

After neglecting exponentially suppressed $\mathcal{O}\left(e^{-L}\right)$ terms, one obtains

$$
\begin{array}{lll}
\mathcal{N}_{A} \approx \frac{\left(-L_{A}+e^{2 / \ell_{b}}\left(L_{A}-2\right)\right)^{\frac{1}{2}}}{\left(e^{2 / \ell_{b}}-1\right)^{\frac{3}{2}}}, & \mathcal{N}_{B} \approx \frac{\left(-L_{B}+e^{2 / \ell_{b}}\left(L_{B}-2\right)\right)^{\frac{1}{2}}}{\left(e^{2 / \ell_{b}}-1\right)^{\frac{3}{2}}}, \\
\mathcal{N}_{A}^{\prime \prime} \approx \frac{e^{1 / \ell_{b}}}{e^{2 / \ell_{b}}-1} e^{L_{A} / \ell_{b}}, & \mathcal{N}_{B}^{\prime \prime} \approx \frac{1}{e^{2 / \ell_{b}}-1} e^{-L_{A} / \ell_{b}}, \\
\mathcal{N}_{A}^{\prime} \approx \frac{e^{1 / \ell_{b}}}{\left(e^{2 / \ell_{b}}-1\right)^{\frac{1}{2}}} e^{L_{A} / \ell_{b}}, & \mathcal{N}_{B}^{\prime} \approx \frac{1}{\left(e^{2 / \ell_{b}}-1\right)^{\frac{1}{2}}} e^{-L_{A} / \ell_{b}} .
\end{array}
$$

The singular values of (B.21), using (B.22), read

$$
\begin{array}{rlrl}
\zeta_{0} & =\frac{\mathcal{N}}{\mathcal{N}} \approx \frac{e^{2 / \ell_{b}}\left(L_{A}-2\right)-L_{A}}{e^{2 / \ell_{b}}(L-2)-L}, & \zeta^{\prime} & =\frac{\mathcal{N}_{A}^{\prime \prime} N_{B}^{\prime}}{N} \approx \frac{e^{2 / \ell_{b}}}{e^{2 / \ell_{b}}(L-2)-L}, \\
\zeta_{0}^{\prime}=\frac{\mathcal{N}_{B}}{\mathcal{N}} \approx \frac{e^{2 / \ell_{b}}\left(L-L_{A}-2\right)-\left(L-L_{A}\right)}{e^{2 / \ell_{b}}(L-2)-L}, & \zeta^{\prime \prime}=\frac{\mathcal{N}_{A}^{\prime} \mathcal{N}_{B}^{\prime \prime}}{\mathcal{N}} \approx \frac{e^{2 / \ell_{b}}}{e^{2 / \ell_{b}}(L-2)-L} .
\end{array}
$$

As for two particles (see section Appendix B.1), $\zeta_{0}$ and $\zeta_{0}^{\prime}$ are finite in the limit $L \rightarrow \infty$, and are bulk-related, whereas $\zeta^{\prime}$ and $\zeta^{\prime \prime}$, which are vanishing, have boundary origin. It can be checked that the corresponding singular vectors are exponentially localized at the boundary of block $A$.

From (B.23) the half-chain entanglement entropy, neglecting terms $\mathcal{O}\left(1 / L^{2}\right)$, reads

$$
S_{A}(L / 2)=\log (2)+2 \frac{-1+\log (2)-\log \left(1-e^{-2 / \ell_{b}}\right)-\log (L)}{1-e^{-2 / \ell_{b}}} \frac{1}{L}+\mathcal{O}\left(1 / L^{2}\right),
$$

and in terms of the bound-state energy $E$ (cf. (27))

$$
S_{A}(L / 2) \approx \log (2)-\frac{3}{2 L E}[1+2 \log (2)+\log (L)+\log (-E)-\log (6)] .
$$

As for the two-particle bound state, the correct expression, which takes into account all the three components in (B.18), is obtained as

$$
S_{A}(L / 2) \approx \log (2)-\frac{6}{2 L E}[1+2 \log (2)+\log (L)+\log (-E)-\log (6)] .
$$

This is checked numerically in Figure 6 (c).

\section{Appendix C. Eigenstates containing a single 2-particle bound state}

In this section we discuss the structure of the Bethe wavefunction (6) for $M$-particle eigenstates of the $X X X$ chain with string content $\{M-2,1\}$. These correspond to solutions of the Bethe-Takahashi equations (20) containing $M-21$-strings and a single 2-string. Physically, the resulting wavefunctions contain magnons and a two-particle bound state. Due to the presence of the 2-string, divergent terms in (7) appear. 
To illustrate the construction of the wavefunction we first focus on the elementary case with $M=3$ (i.e., string content $\{2,1\}$ ), considering $\Lambda=\left\{\lambda_{1}, \lambda_{2}, \lambda_{3}\right\}$ roots of the Bethe equations (12) of the form

$$
\Lambda=\left\{\begin{array}{l}
\lambda_{1}=\lambda \\
\lambda_{2}=\lambda^{\prime}+i+i \delta \\
\lambda_{3}=\lambda^{\prime}-i-i \delta
\end{array}\right.
$$

Notice that we assume $\operatorname{Re}\left(\lambda_{2}\right)=\operatorname{Re}\left(\lambda_{3}\right) \neq \operatorname{Re}\left(\lambda_{1}\right)$. Using (29) one obtains that the only diverging scattering phases are $\theta_{3,2}=-\theta_{2,3} \rightarrow-i \log (\delta)$. It is straightforward, using the Bethe equations (10), to derive the relation

$$
\theta_{3,2}=\theta_{2,3}+\theta_{1,3}-\theta_{1,2}-\left(k_{2}-k_{3}\right) \frac{L}{2} \bmod \pi .
$$

Using (C.2) the Bethe wavefunction (6) reduces to

$$
\begin{aligned}
\left|\Psi_{\{2,1\}}\right\rangle \propto \exp \left(i \theta_{2,3}\right) \sum_{x_{1}<x_{2}<x_{3}}\{ & \\
& \exp \left[i K_{b}\left(x_{2}+x_{3}\right)+i k_{1} x_{1}+\theta_{1,2}+\theta_{1,3}-\left(x_{3}-x_{2}\right) / \ell_{b}\right]+ \\
& \exp \left[i K_{b}\left(x_{1}+x_{3}\right)+i k_{1} x_{2}+\theta_{2,1}+\theta_{1,3}-\left(x_{3}-x_{1}\right) / \ell_{b}\right]+ \\
& \exp \left[i K_{b}\left(x_{2}+x_{3}\right)+i k_{1} x_{3}+\theta_{2,1}+\theta_{3,1}-\left(x_{2}-x_{1}\right) / \ell_{b}\right]+ \\
& \exp \left[i K_{b}\left(x_{2}+x_{3}\right)+i k_{1} x_{1}+2 \theta_{1,3}+\theta_{1,2}-\left(x_{2}-x_{3}+L\right) / \ell_{b}\right]+ \\
& \exp \left[i K_{b}\left(x_{1}+x_{2}\right)+k_{1} x_{3}+2 \theta_{2,1}-\left(x_{1}-x_{2}+L\right) / \ell_{b}\right]+ \\
& \left.\exp \left[i K_{b}\left(x_{1}+x_{3}\right)+i k_{1} x_{2}-\left(x_{1}-x_{3}+L\right) / \ell_{b}\right]\right\}\left|x_{1}, x_{2}, x_{3}\right\rangle,
\end{aligned}
$$

where $K_{b} \equiv k_{2}+k_{3} \in \mathbb{R}, \ell_{b}$ is the bound-state length (cf. (27)), and the subscript in $\left|\Psi_{\{2,1\}}\right\rangle$ is to stress that we are considering string content $\{2,1\}$. Notice the divergent factor $\exp \left(i \theta_{2,3}\right)$ in (C.3).

A similar procedure can be used in the generic case with $M$ particles and string content $\{M-2,1\}$. First, the generalization of (C.2), assuming that $\lambda_{M-1}, \lambda_{M}$ form a 2-string, is obtained as

$$
\theta_{M, M-1}=\theta_{M-1, M}-\left(k_{M-1}-k_{M}\right) \frac{L}{2}-\sum_{j<M-1}\left(\theta_{j, M-1}+\theta_{j, M}\right) \quad \bmod \pi .
$$

The Bethe wavefunction is obtained from the general expression (6) substituting every occurrence of $\theta_{M-1, M}$ using (C.4).

\section{Appendix D. A semiclassical upper bound for the entanglement entropy}

In this section we derive the upper bound (5) for the entanglement entropy.

We start considering a bipartition of a chain of length $L$ into two parts $A$ and $B$ of length $L_{A}$ and $L_{B}$, respectively. Here we define the ratio $\omega$ as $\omega \equiv L_{A} / L$. Given the generic eigenstate (6) of the $X X X$ chain in the sector with $M$ particles, the Schmidt matrix $\mathbb{M}(A \mid B)$ (cf. (A.3) for the definition) exhibits a block structure, each block $\mathbb{M}^{(k)}(A \mid B)$ corresponding to a different number of particles $k$ in subsystem $A$. 
The number of non-zero singular values in $\mathbb{M}^{(k)}(A \mid B)$ is given as the number of ways of assigning $k$ quasi-momenta from the set $\left\{k_{1}, k_{2}, \ldots, k_{M}\right\}$ (cf. (6)) to the $k$ particles in $A$. This is given by the binomial coefficient $C(M, k) \equiv M ! /(k !(M-k) !)$.

Assuming that every particle with given momentum is fully delocalized in the chain ("semiclassical" approximation), the singular values $\left\{\zeta_{i}\right\}$ with $i=1,2 \ldots, C(M, k)$ in $\mathbb{M}^{(k)}(A \mid B)$ are all degenerate, and are given as $\zeta_{i}=\left(\omega^{k}(1-\omega)^{M-k}\right)^{1 / 2} \forall i$. Notice that $\left\{\zeta_{i}^{2}\right\}$, which would be the eigenvalues of the reduced density matrix $\rho_{A}$ for block $A$, correspond to the semiclassical probabilities of finding $k$ particles in $A$ and $M-k$ in $B$. Finally, the upper bound $S_{M A X}\left(M, L_{A}\right)$ for the entanglement entropy is given as

$$
S_{M A X}\left(M, L_{A}\right)=-\sum_{k=0}^{M} \frac{M !}{k !(M-k) !} \omega^{k}(1-\omega)^{M-k} \log \left[\omega^{k}(1-\omega)^{M-k}\right] .
$$

It is convenient to consider the large $M$ limit. Thus, approximating the binomial coefficient with a gaussian, one obtains

$S_{M A X}\left(M, L_{A}\right)=-\frac{1}{\sqrt{2 \pi M \omega(1-\omega)}} \sum_{k=0}^{M} e^{-\frac{(k-M \omega)^{2}}{2 M \omega(1-\omega)}}(k \log \omega+(M-k) \log (1-\omega))$.

Turning the sum over $k$ into an integral, and taking the integration in the full interval $k \in(-\infty, \infty)$ (instead of $[0, \infty)),(\mathrm{D} .2)$ becomes

$$
S_{M A X}\left(M, L_{A}\right)=-M[\omega \log \omega+(1-\omega) \log (1-\omega)] .
$$

Interestingly, $S_{M A X}\left(M, L_{A}\right)$ is proportional to the total number of particles in the system. Notice the symmetry under the exchange of the two subsystems $L_{A} \leftrightarrow L_{B}$, i.e., $\omega \leftrightarrow 1-\omega$.

\section{References}

[1] L. Amico, R. Fazio, A. Osterloh, and V. Vedral, Entanglement in Many-Body Systems, Rev. Mod. Phys. 80, 517 (2008).

[2] J. Eisert, M. Cramer, and M. B. Plenio, Area laws for the entanglement entropy - a review, Rev. Mod. Phys. 82, 277 (2009).

[3] P. Calabrese, J. Cardy, and B. Doyon Eds., Special issue: Entanglement entropy in extended systems, J. Phys. A 42, 50 (2009).

[4] P. Calabrese and J. Cardy, Entanglement entropy and conformal field theory, J. Phys. A 42504005 (2009).

[5] M. Kaulke and I. Peschel, A DMRG study of the q-symmetric Heisenberg chain, Eur. Phys. J. B 5, 727 (1998).

[6] H. Li, and F. D. M. Haldane, Entanglement Spectrum as a Generalization of Entanglement Entropy: Identification of Topological Order in Non-Abelian Fractional Quantum Hall Effect States, Phys. Rev. Lett. 101, 010504 (2008).

[7] P. Calabrese, A. Lefevre, Entanglement spectrum in one-dimensional systems, Phys. Rev. A 78, 032329 (2008).

[8] N. Regnault, B. A. Bernevig, F. D. M. Haldane, Topological Entanglement and Clustering of Jain Hierarchy States, Phys. Rev. Lett. 103, 016801 (2009).

[9] B. Nienhuis, M. Campostrini, and P. Calabrese, Entanglement, combinatorics and finite-size effects in spin-chains, J. Stat. Mech. (2009) P02063. 
[10] N. Bray-Ali, L. Ding, and S. Haas, Topological order in paired states of fermions in two dimensions with breaking of parity and time-reversal symmetries, Phys. Rev. B 80, 180504(R) (2009).

[11] L. Fidkowski, Entanglement Spectrum of Topological Insulators and Superconductors, Phys. Rev. Lett. 104, 130502 (2010).

[12] A. M. Läuchli, E. J. Bergholtz, J. Suorsa, and M. Haque, Disentangling Entanglement Spectra of Fractional Quantum Hall States on Torus Geometries, Phys. Rev. Lett. 104, 156404 (2010).

[13] R. Thomale, A. Sterdyniak, N. Regnault, and B. A. Bernevig, Entanglement Gap and a New Principle of Adiabatic Continuity, Phys. Rev. Lett. 104, 180502 (2010).

[14] H. Yao and X. L. Qi, Entanglement Entropy and Entanglement Spectrum of the Kitaev Model, Phys. Rev. Lett. 105, 080501 (2010).

[15] E. Prodan, T. L. Hughes, and B. A. Bernevig, Entanglement Spectrum of a Disordered Topological Chern Insulator, Phys. Rev. Lett. 105, 115501 (2010).

[16] F. Pollmann, A. M. Turner, E. Berg, M. Oshikawa, Entanglement spectrum of a topological phase in one dimension, Phys. Rev. B 81, 064439 (2010).

[17] M. Kargarian and G. A. Fiete, Topological phases and phase transitions on the square-octagon lattice, Phys. Rev. B 82, 085106 (2010).

[18] A. M. Turner, Y. Zhang, A. Vishwanath, Entanglement and inversion symmetry in topological insulators, Phys. Rev. B 82, 241102R (2010).

[19] Z. Papic, B. A. Bernevig, and N. Regnault, Topological Entanglement in Abelian and Non-Abelian Excitation Eigenstates, Phys. Rev. Lett. 106, 056801 (2011).

[20] L. Fidkowski, T. S. Jackson and I. Klich, Model Characterization of Gapless Edge Modes of Topological Insulators Using Intermediate Brillouin-Zone Functions, Phys. Rev. Lett. 107, 036601 (2011).

[21] X. Deng and L. Santos, Entanglement spectrum of one-dimensional extended Bose-Hubbard models, Phys. Rev. B 84, 085138 (2011).

[22] J. Dubail, and N. Read, Entanglement Spectra of Complex Paired Superfluids, Phys. Rev. Lett. 107, 157001 (2011).

[23] J. Schliemann, Entanglement spectrum and entanglement thermodynamics of quantum Hall bilayers at $\nu=1$, Phys. Rev. B 83, 115322 (2011).

[24] T. L. Hughes, E. Prodan, B. A. Bernevig, Inversion-symmetric topological insulators, Phys. Rev. B 83, 245132 (2011).

[25] N. Regnault and B. A. Bernevig, Fractional Chern Insulator, Phys. Rev. X 1, 021014 (2011).

[26] V. Alba, M. Haque and A. M. Läuchli, Boundary-Locality and Perturbative Structure of Entanglement Spectra in Gapped Systems, Phys. Rev. Lett. 108, 227201 (2012).

[27] X. L. Qi, H. Katsura, and A. W. W. Ludwig, General Relationship between the Entanglement Spectrum and the Edge State Spectrum of Topological Quantum States, Phys. Rev. Lett. 108, 196402 (2012).

[28] R. Lundgren, V. Chua, and G. Fiete, Entanglement Entropy and Spectra of the One-dimensional Kugel-Khomskii Model, Phys. Rev. B 86, 224422 (2012).

[29] D. Poilblanc, N. Schuch, D. Perez-Garcia, and J.I. Cirac, Topological and entanglement properties of resonating valence bond wave functions, Phys. Rev. B 86, 014404 (2012).

[30] G. De Chiara, L. Lepori, M. Lewenstein, and A. Sanpera, Entanglement Spectrum, Critical Exponents, and Order Parameters in Quantum Spin Chains, Phys. Rev. Lett. 109, 237208 (2012).

[31] V. Alba, M. Haque, and A. M. Läuchli, Entanglement spectrum of the Heisenberg XXZ chain near the ferromagnetic point, J. Stat. Mech. (2012) P08011.

[32] L. Lepori, G. De Chiara, and A. Sanpera, Entanglement Spectrum, Critical Exponents, and Order Parameters in Quantum Spin Chains, Phys. Rev. B 87235107 (2013).

[33] A. M. Läuchli, Operator content of real-space entanglement spectra at conformal critical points, arXiv:1303.0741 (2013) unpublished.

[34] C.-M. Chung, L. Bonnes, P. Chen, and A. Läuchli, Entanglement spectroscopy using Quantum 
Monte Carlo, Phys. Rev. B 89, 195147 (2014).

[35] R. Lundgren, Y. Fuji, S. Furukawa, and M. Oshikawa, Entanglement spectra between coupled Tomonaga-Luttinger liquids: Applications to ladder systems and topological phases, Phys. Rev. B 88, 245137 (2013).

[36] F. Kolley, S. Depenbrock, I. P. McCulloch, U. Schollwöck, and V. Alba, Entanglement spectroscopy of SU(2)-broken phases in two dimensions, Phys. Rev. B 88, 144426 (2013).

[37] A. Chandran, V. Khemani, and S. L. Sondhi, How universal is the entanglement spectrum?, arXiv:13112964.

[38] T. Grover, Entanglement of Interacting Fermions in Quantum Monte Carlo Calculations, Phys. Rev. Lett. 111, 130402 (2013).

[39] F. F. Assaad, T. C. Lang, and F. Parisen Toldin, Entanglement Spectra of Interacting Fermions in Quantum Monte Carlo simulations, Phys. Rev. B 89, 125121 (2013).

[40] A. Petrescu, H. F. Song, S. Rachel, Z. Ristivojevic, C. Flindt, N. Laflorencie, I. Klich, N. Regnault, and K. Le Hur, Fluctuations and Entanglement spectrum in quantum Hall states, arXiv:1405.7816 (2014).

[41] D. J. Luitz, X. Plat, N. Laflorencie, and F. Alet, Improving entanglement and thermodynamic Rényi entropy measurements in Quantum Monte Carlo, arXiv:1405.7391.

[42] D. J. Luitz, N. Laflorencie, and F. Alet, Participation spectroscopy and entanglement Hamiltonian of quantum spin models, arXiv:1404.3717.

[43] D. J. Luitz, F. Alet, and N. Laflorencie, Shannon entropy and participation spectra across the $3 d$ $O(3)$ criticality, Phys. Rev. B 89, 165106 (2014).

[44] R. Lundgren, J. Blair, M. Greiter, A. Läuchli, G. Fiete, and R. Thomale, Momentum Space Entanglement Spectrum of Bosons and Fermions with Interactions, arXiv:1404.7545.

[45] J. Schliemann, Entanglement thermodynamics, arXiv:1405.2150.

[46] M. Udagawa, Y. Motome, Entanglement Spectrum in Cluster Dynamical Mean-Field Theory, arXiv:14065960.

[47] C. Holzhey, F. Larsen, and F. Wilczek, Geometric and Renormalized Entropy in Conformal Field Theory, Nucl. Phys. B 424, 443 (1994).

[48] G. Vidal, J. I. Latorre, E. Rico, and A. Kitaev, Entanglement in quantum critical phenomena, Phys. Rev. Lett. 90, 227902 (2003). J. I. Latorre, E. Rico, and G. Vidal, Ground state entanglement in quantum spin chains, Quant. Inf. and Comp. 4, 048 (2004).

[49] P. Calabrese and J. Cardy, Entanglement entropy and quantum field theory, J. Stat. Mech. (2004) P06002. P. Calabrese and J. Cardy, Entanglement entropy and quantum field theory: a nontechnical introduction, Int. J. Quant. Inf. 4, 429 (2006).

[50] S. Furukawa, V. Pasquier, and J. Shiraishi, Mutual information and compactification radius in a $\mathrm{c}=1$ critical phase in one dimension, Phys. Rev. Lett. 102, 170602 (2009).

[51] M. Caraglio and F. Gliozzi, Entanglement entropy and twist fields, JHEP 0811: 076 (2008).

[52] P. Calabrese, J. Cardy, and E. Tonni, Entanglement entropy of two disjoint intervals in conformal field theory, J. Stat. Mech. P11001 (2009).

[53] F. Igloi and I. Peschel, On reduced density matrices for disjoint subsystems, EPL 8940001 (2010).

[54] M. Fagotti and P. Calabrese, Entanglement entropy of two disjoint blocks in XY chains, J. Stat. Mech. (2010) P04016.

[55] P. Calabrese, J. Cardy, and E. Tonni, Entanglement entropy of two disjoint intervals in conformal field theory II, J. Stat. Mech. (2011) P01021.

[56] H. Casini and M. Huerta, A finite entanglement entropy and the c-theorem, Phys. Lett. B 600142 (2004). H. Casini, C. D. Fosco, and M. Huerta, Entanglement and alpha entropies for a massive Dirac field in two dimensions, J. Stat. Mech. P05007 (2005). H. Casini and M. Huerta, Remarks on the entanglement entropy for disconnected regions, JHEP 0903: 048 (2009). H. Casini and M. Huerta, Reduced density matrix and internal dynamics for multicomponent regions, Class. Quant. Grav. 26, 185005 (2009). H. Casini, Entropy inequalities from reflection positivity, J. Stat. Mech. P08019 (2010). D. D. Blanco, H. Casini, Entanglement entropy for non-coplanar 
regions in quantum field theory, Class. Quant. Grav. 28: 215015 (2011).

[57] P. Facchi, G. Florio, C. Invernizzi, and S. Pascazio, Entanglement of two blocks of spins in the critical Ising model, Phys. Rev. A 78, 052302 (2008).

[58] S. Ryu and T. Takayanagi, Holographic derivation of entanglement entropy from AdS/CFT, Phys. Rev. Lett. 96, 181602 (2006). S. Ryu and T. Takayanagi, Aspects of holographic entanglement entropy, JHEP 0608: 045 (2006). V. E. Hubeny and M. Rangamani, Holographic entanglement entropy for disconnected regions, JHEP 0803: 006 (2008). M. Headrick and T. Takayanagi, A holographic proof of the strong subadditivity of entanglement entropy, Phys. Rev. D 76, 106013 (2007). T. Nishioka, S. Ryu, and T. Takayanagi, Holographic entanglement entropy: an overview, J. Phys. A 42504008 (2009). E. Tonni, Holographic entanglement entropy: near horizon geometry and disconnected regions, JHEP 1105: 004 (2011). A. Allais, E. Tonni, Holographic evolution of the mutual information, JHEP 1201: 102 (2012).

[59] V. Alba, L. Tagliacozzo, and P. Calabrese, Entanglement entropy of two disjoint blocks in critical Ising models, Phys. Rev. B 81, 060411 (2010).

[60] V. Alba, L. Tagliacozzo, and P. Calabrese, Entanglement entropy of two disjoint intervals in critical $\mathrm{c}=1$ theories, J. Stat. Mech. (2011) P06012.

[61] M. Fagotti, New insights in the entanglement of two disjoint blocks, EPL 9717007 (2012).

[62] A. Coser, L. Tagliacozzo, and E. Tonni, On Rényi entropies of disjoint intervals in conformal field theory, J. Stat. Mech. (2014) P01008.

[63] P. Calabrese, M. Campostrini, F. H. L. Essler, and B. Nienhuis, Parity effects in the scaling of block entanglement in gapless spin chains, Phys. Rev. Lett. 104, 095701 (2010).

[64] P. Calabrese and F. H. L. Essler, Universal corrections to scaling for block entanglement in spin-1/2 XX chains, J. Stat. Mech. (2010) P08029.

[65] J. Cardy and P. Calabrese, Unusual corrections to scaling in entanglement entropy, J. Stat. Mech. (2010) P04023.

[66] P. Calabrese, J. Cardy, and I. Peschel, Corrections to scaling for block entanglement in massive spin-chains, J. Stat. Mech. (2010) P09003.

[67] M. Fagotti and P. Calabrese, Universal parity effects in the entanglement entropy of XX chains with open boundary conditions, J. Stat. Mech. (2011) P01017.

[68] J. C. Xavier and F. C. Alcaraz, Rényi Entropy and Parity Effect of the Anisotropic Spin-s Heisenberg Chains with a Magnetic Field, Phys. Rev. B 83, 214425 (2011).

[69] P. Calabrese, M. Mintchev, and E. Vicari, The entanglement entropy of 1D systems in continuous and homogenous space, J. Stat. Mech. (2011) P09028.

[70] V. Alba, M. Fagotti, and P. Calabrese, Entanglement entropy of excited states, J. Stat. Mech. (2009) P10020.

[71] F. C. Alcaraz, M. I. Berganza, and G. Sierra, Entanglement of low-energy excitations in conformal field theory, Phys. Rev. Lett. 106, 201601 (2011).

[72] I. Pizorn, Universality in entanglement of quasiparticle excitations, arXiv:1202.3336.

[73] M. I. Berganza, F. C. Alcaraz, and G. Sierra, Entanglement of excited states in critical spin chains, J. Stat. Mech. (2012) P01016.

[74] G. Wong, I. Klich, L. A. P. Zayas, and D. Vaman, Entanglement Temperature and Entanglement Entropy of Excited States, JHEP 12 (2013) 020.

[75] M. Storms, and R. R. P. Singh, Entanglement in ground and excited states of gapped fermion systems and their relationship with fermi surface and thermodynamic equilibrium properties, Phys. Rev. E 89, 012125 (2014).

[76] R. Berkovits, Two-particle excited states entanglement entropy in a one-dimensional ring, Phys. Rev. B 87, 075141 (2013).

[77] F. H. L. Essler, A. M. Läuchli, and P. Calabrese, Shell-Filling Effect in the Entanglement Entropies of Spinful Fermions, Phys. Rev. Lett. 110, 115701 (2013).

[78] M. Nozaki, T. Numasawa, T. Takayanagi, Quantum Entanglement of Local Operators in Conformal Field Theories, Phys. Rev. Lett. 112, 111602 (2014). 
[79] G. Ramirez, J. Rodriguez-Laguna, and G. Sierra, Entanglement in low-energy states of the randomhopping model, arXiv:1402.5015.

[80] F. Ares, J. G. Esteve, F. Falceto, and E. Sánchez-Burillo, Excited states entanglement in homogeneous fermionic chains, arXiv:1401.5922.

[81] Y. Huang, and J. Moore, Excited-state entanglement and thermal mutual information in random spin chains, arXiv:1405.1817.

[82] T. Pálmai, Excited state entanglement in conformal field theory: extensivity and the role of microscopic details, arXiv:1406.3182.

[83] E. Lieb, T. Schultz, and D. Mattis, Ann. Phys. 16, 407 (1961).

[84] E. Barouch, B. M. McCoy, and M. Dresden, Phys. Rev. A 2, 1075 (1970).

[85] E. Barouch and B. McCoy, Phys. Rev. A 3, 786 (1971).

[86] E. Barouch and B. M. McCoy, Phys. Rev. A 3, 2137 (1971).

[87] B. M. McCoy, E. Barouch, and D. B. Abraham, Phys. Rev. A 4, 2331 (1971).

[88] V. E. Korepin, N. M. Bogoliubov, A. G. Izergin, Quantum Inverse Scattering Method and Correlation Functions, Cambridge University Press, Cambridge, 1997.

[89] P. Barmettler, M. Punk, V. Gritsev, E. Demler, and E. Altman, Quantum quenches in the anisotropic spin-1/2 Heisenberg chain: different approaches to many-body dynamics far from equilibrium, New J. Phys. 12, 055017 (2010).

[90] V. Gritsev, T. Rostunov, and E. Demler, Exact methods in analysis of nonequilibrium dynamics of integrable models: application to the study of correlation function in nonequilibrium $1 D$ Bose gas, J. Stat. Mech. (2010) P05012.

[91] G. Vidal, Efficient classical simulation of one-dimensional quantum many-body systems, Phys. Rev. Lett. 93, 040502 (2004).

[92] A. J. Daley, C. Kollath, U. Schollwöck, and G. Vidal, Time-dependent density-matrix renormalization-group using adaptive effective Hilbert spaces, J. Stat. Mech. (2004) P04005.

[93] S. White and A. Feiguin, Real-Time Evolution Using the Density Matrix Renormalization Group, Phys. Rev. Lett. 93, 076401 (2004).

[94] U. Schollwöck, The density-matrix renormalization group, Rev. Mod. Phys. 77, 259 (2005).

[95] U. Schollwöck, The density-matrix renormalization group in the age of matrix product states, Annals of Physics 326, 96 (2011).

[96] H. Bethe, Zur Theorie der Metalle. I. Eigenwerte und Eigenfunktionen der linearen Atomkette, Z. Phys. 71, 205 (1931).

[97] M. Takahashi, Thermodynamics of one-dimensional solvable models, Cambridge University Press, Cambridge, 1999.

[98] J. Hanus, Bound states in the Heisenberg ferromagnet, Phys. Rev. Lett. 11, 336 (1963).

[99] M. Wortis, Bound states of two spin waves in the Heisenberg ferromagnet, Phys. Rev. 132, 85 (1963).

[100] H. C. Fogedby, The spectrum of continuous isotropic quantum Heisenberg chain: quantum solitons as magnon bound states, J. Phys. C 13, L195 (1980).

[101] T. Schneider, Solitons and magnon bound states in ferromagnetic Heisenberg chains, Phys. Rev. B 24, 5327 (1981).

[102] M. Kohno, Dynamically Dominant Excitations of String Solutions in the Spin-1/2 Antiferromagnetic Heisenberg Chain in a Magnetic Field, Phys. Rev. Lett. 102, 037203 (2009).

[103] M. Ganahl, E. Rabel, F. H. L. Essler, H. G. Evertz, Observing complex bound states in the spin-1/2 Heisenberg XXZ chain, Phys. Rev. Lett. 108077206 (2012).

[104] E. Haller, M. Gustavsson, M. J. Mark, J. G. Danzl, R. Hart, G. Pupillo, and H-C. Nägerl, Relaization of an Excited, Strongly Correlated Quantum Gas Phase, Science 325, 1224 (2009).

[105] T. Fukuhara, P. Schauss, M. Endres, S. Hild, M. Cheneau, I. Bloch, and C. Gross, Microscopic observation of magnon bound states and their dynamics, Nature 502, 76 (2013).

[106] V. Popkov and M. Salerno, Logarithmic divergence of the block entanglement entropy for the ferromagnetic Heisenberg model, Phys. Rev. A 71, 012301 (2005). 
[107] V. Popkov and M. Salerno, Reduced-density-matrix spectrum and block entropy of permutationally invariant many-body systems, Phys. Rev. E 82, 011142 (2010).

[108] O. A. Castro-Alvaredo and B. Doyon, Permutation operators, entanglement entropy, and the XXZ spin chain in the limit $\Delta \rightarrow-1$, J. Stat. Mech. (2011) P02001.

[109] O. A. Castro-Alvaredo and B. Doyon, Entanglement entropy of highly degenerate states and fractal dimensions, Phys. Rev. Lett. 108, 10401 (2012).

[110] M. Karbach, G. Muller, Introduction to the Bethe ansatz I, Computers in Physics 11, 36 (1997). M. Karbach, K. Hu, and G. Muller, Introduction to the Bethe ansatz II, Computers in Physics 12, 565 (1998). M. Karbach, K. Hu, and G. Muller, Introduction to the Bethe ansatz III, arxiv:0008018.

[111] C. N. Yang, and C. P. Yang, One-Dimensional Chain of Anisotropic Spin-Spin Interactions. I. Proof of Bethe's Hypothesis for Ground State in a Finite System, Phys. Rev. 150, 321 (1966).

[112] F. H. L. Essler, V. E. Korepin, and K. Schoutens, Fine structure of the Bethe ansatz for the spin- $\frac{1}{2}$ Heisenberg $X X X$ model, J. Phys. A: Math. Theor. 254115 (1992).

[113] R. Hagemans and J.-S. Caux, Deformed strings in the Heisenberg model, J. Phys. A: Math. Theor. 4014605 (2007).

[114] V. Alba, K. Saha, and M. Haque, Bethe ansatz description of edge-localization in the openboundary $X X Z$ spin chain, J. Stat. Mech. (2013) P10018.

[115] H. J. de Vega and F. Woynarovich, Method for calculating finite size corrections in Bethe ansatz systems: Heisenberg chain and six-vertex model, Nucl. Phys. B 251, 439 (1985).

[116] F. C. Alcaraz, M. N. Barber, and M. T. Batchelor, Conformal invariance and the spectrum of the $X X Z$ chain, Phys. Rev. Lett. 58, 771 (1987).

[117] H. J. de Vega, Finite-size corrections for nested Bethe ansatz models and conformal invariance J. Phys. A: Math. Gen. 20, 6023 (1987).

[118] F. C. Alcaraz and M. J. Martins, Conformal invariance and critical exponents of the TakhtajanBabujian modelsJ. Phys. A: Math. Gen. 21, 4397 (1988).

[119] C. Destri and H. J. de Vega, New thermodynamic Bethe ansatz equations without strings, Phys. Rev. Lett. 69, 2313 (1992).

[120] A. A. Vladimirov, Proof of the invariance of the Bethe-ansatz solutions under complex conjugation, Teor. Mat. Fiz. 66, 154 (1986).

[121] D. Biegel, M. Karbach, G. Müller, K. Wiele, Spectrum and transition rates of the $X X$ chain analyzed via Bethe ansatz, Phys. Rev. B 69, 174404 (2004).

[122] S.-J. Gu, N. M. R. Peres, and Y.-Q. Li, Numerical and Monte Carlo Bethe ansatz method: 1D Heisenberg model, Eur. Phys. J. B 48, 157 (2005). 